\title{
Diffusion of charged particles in tokamak-like stochastic magnetic and electric fields
}

\author{
M. Coronado and J. Vitela E. \\ Instituto de Ciencias Nucleares, Universidad Nacional Autónoma de México, A. Postal 70-543, \\ 04510 México, D.F. \\ A. Z. Akcasu \\ Department of Nuclear Engineering, The University of Michigan, Ann Arbor, Michigan 48109
}

(Received 24 March 1992; accepted 11 August 1992)

In this paper the diffusion of guiding centers induced by stochastic magnetic and electric field fluctuations, with both time and space dependence, is analyzed for the case of tokamak plasmas. General experimental results on tokamak fluctuations are used to derive guidingcenter equations that properly describe the particle motion. These equations assume uniform average magnetic and electric fields with random stationary Gaussian fluctuations that constitute a homogeneous and cylindrically symmetric turbulence. By applying Novikov's theorem, a Fokker-Planck equation for the probability distribution function is derived and an expression for the guiding-center diffusion coefficient is obtained. This coefficient not only contains the standard terms due to the stochastic wandering of the magnetic lines and the stochastic electric drift, but also new terms due to the stochastic curvature and $\nabla B$ drifts. The form of these terms is shown explicitly in terms of the correlation functions of the fields.

\section{INTRODUCTION}

At present, one of the most important problems in tokamak research is anomalous transport, which, in general, seems to be caused by turbulent processes occurring in the plasma. Although strong efforts have been made during the past decade to measure the statistical properties of the stochastic fluctuations in particle density, temperature, electric potential, magnetic field, and electric current in tokamaks, ${ }^{1-3}$ a complete self-consistent model describing plasma turbulence and anomalous transport is, due to its complexity, still lacking. This complete description requires a simultaneous solution to Maxwell's equations and the kinetic or fluid equations. The theoretical approaches used to treat plasma turbulence and anomalous transport have been described by Haas and Thyagaraja ${ }^{4}$ and classified as microstability, fluid, and test-particle theories. Research trends have been focused on two complementary areas: one is the study of plasma instabilities that might give rise to the kind of turbulence found in tokamaks ( $d y$ namics), and the second is the analysis of the transport induced by externally given stochastic fluctuations of the type seen in tokamak plasmas (energetics). Two basic mechanisms of radial transport are generally recognized: ${ }^{5}$ low-frequency electric fluctuations that produce stochastic electric drifts, and magnetic field microfluctuations that lead to a weak destruction of the magnetic surfaces. Singleparticle theories are intrinsically non-self-consistent, but do provide a useful tool to analyze the basic physics involved in particle transport.

The guiding-center approach is a single-particle technique extensively used to treat problems involving one or both of the mechanisms of anomalous transport mentioned above. These problems contain among many others, issues such as: the diffusion of charged particles moving along stochastic magnetic field lines with weakly destroyed magnetic surfaces; ${ }^{6-9}$ the transition of the particle motion from deterministic to stochastic in a homogeneous magnetic field due to the presence of electrostatic waves; ${ }^{10}$ the diffusion of charged particles in a uniform magnetic field with stochastic electrostatic fluctuations; ${ }^{11}$ the particle transport in a toroidal magnetic field with time-dependent electric and magnetic fluctuations; ${ }^{12}$ the diffusion of circulating and trapped particles in a toroidal magnetic field due to time-dependent stochastic electromagnetic fluctuations, ${ }^{13}$ etc. However, the transport induced by stochastic $\nabla B$ and curvature drifts has not received much attention, since they are believed to be ncgligible compared with the transport in weakly destroyed magnetic surfaces or to the diffusion due to the stochastic electric drifts. This point is questionable for the case of magnetic fluctuations with large wave numbers, where strong magnetic gradients might be present, and the subject, therefore requires further research. In this paper we develop an approach to diffusion induced by stochastic fluctuations with time and space dependence taking into account the above-mentioned magnetic drifts. The inclusion of a space dependence of the fluctuations in an analytical treatment is a serious mathematical problem since the stochastic force on the particle depends on the position of the particle. The approach we develop here to deal with space-dependent fluctuations is based on the application of Novikov's theorem, which holds for Gaussian stochastic fluctuations, and gives results that are valid in the so-called quasilinear limit. ${ }^{9}$ In our formulation, we take the available experimental information to analyze the relative magnitudes of terms in the guiding center equations with fluctuations and retain the lowest-order terms of the $\nabla B$ and curvature drifts. To keep the mathematical problem within reasonable limits, we 
consider an average magnetic field that is uniform and we assume Gaussian homogeneous cylindrically symmetric turbulent electric and magnetic fields, which are prescribed externally. The assumption of homogeneous and cylindrically symmetric turbulence might adequately describe a tokamak when the correlation lengths of the fluctuations are much smaller than the system characteristic lengths. The assumption of fluctuation magnitudes given by a Gaussian probability function is an issue to be tested experimentally. Our calculations reproduce earlier results concerning the diffusion of charged particles in stochastic electric fields, ${ }^{5}$ in weakly destroyed magnetic surfaces, ${ }^{6-9}$ and provide expressions for the diffusion coefficients due to the $\nabla B$ and curvature drifts.

In this work we also include a constant parallel electric field to model the tokamak loop voltage; the collisional friction balances the force produced by this electric field and determines the average parallel velocity of the guiding centers. As we shall see, the radial diffusion due to the stochastic curvature drift depends strongly on the magnitude of the parallel velocity.

A key question in this formulation concerns the ambipolarity of the diffusion which, in a self-consistent treatment, should hold automatically (with account of the relevant anomalous and neoclassical transport mechanisms). However, by using approximation methods, the coupling of the plasma with the fields is usually removed or not taken fully into account, which in general leads to nonintrinsic ambipolarity. This, therefore, should be imposed externally. In this paper we prescribe the electromagnetic fluctuations and consequently obtain nonintrinsically ambipolar diffusion.

It is worthwhile to mention here some valuable efforts by Thyagaraja and Haas ${ }^{14}$ to formulate a semi-selfconsistent numerical approach to anomalous tranport. They employ parallel kinetic equations for ions and electrons, quasineutrality of the fluctuations and the Maxwell equations to obtain density and electric potential fluctuations and heat and particle radial fluxes in terms of prescribed radial magnetic fluctuations in a periodic cylindrical geometry. In this formulation the electric charge is conserved locally and hence, the resulting anomalous diffusion is intrinsically ambipolar.

The paper is organized as follows: In Sec. II we derive the guiding-center equations describing the motion of charged particles in a magnetic field with time- and spacedependent tokamak-like electromagnetic fluctuations. We take a Langevin equation for the parallel velocity and include a constant electric field along the average magnetic field. In Sec. III we apply the equations to uniform magnetic and electric fields, and further, in Sec. IV, we treat the case of fluctuations without space dependence; the model leads to the phenomenon of stochastic heating and diffusion in configuration space. The exact results obtained in this section are used as a benchmark to analyze general results where both space and time dependence are involved. Section $\mathrm{V}$ develops an iterative technique based on Novikov's formula to obtain a Fokker-Planck-like equation for the probability density function of finding the po- sition of a guiding center around a certain point at a given time. The diffusion coefficient is derived in Sec. VI using this Fokker-Planck equation for the case of stationary homogeneous and cylindrically symmetric turbulent electric and magnetic fields. In Sec. VII we present a summary and the concluding remarks. Appendix A contains the details of the calculations of Sec. III, and finally Appendix B discusses some basic properties of the stationary, homogeneous cylindrically symmetric turbulence.

\section{GUIDING-CENTER EQUATIONS WITH TOKAMAK- LIKE ELECTROMAGNETIC FLUCTUATIONS}

The analysis of the transport of charged test particles induced by fluctuations of the type observed in tokamaks starts with the standard guiding-center equations for the position $\mathbf{R}(t)$, the parallel velocity $V_{\|}(t)$, and the perpendicular velocity $V_{1}(t)$ of the guiding center of a test particle. ${ }^{15,16}$ In the nonrelativistic case they are given by

$$
\begin{aligned}
\frac{d \mathbf{R}}{d t}= & V_{\|} \hat{\tau}_{0}+c \frac{\mathbf{E} \times \hat{\tau}_{0}}{B}+\frac{m V_{1}^{2} c}{2 q B} \frac{\hat{\tau}_{0} \times \nabla B}{B}+\frac{m V_{\|}^{2} c}{q B} \hat{\tau}_{0} \\
& \times\left[\left(\hat{\tau}_{0} \cdot \nabla\right) \hat{\tau}_{0}\right]+\frac{m V_{\| c} c}{q B}\left(\hat{\tau}_{0} \times \frac{\partial \hat{\tau}_{0}}{\partial t}\right), \\
\frac{d V_{\|}}{d t}= & \frac{q}{m} \mathbf{E} \cdot \hat{\tau}_{0}+\frac{1}{2} V_{1}^{2}\left(\nabla \cdot \hat{\tau}_{0}\right), \\
\frac{d V_{1}}{d t}= & -\frac{1}{2} V_{1} V_{\|}\left(\nabla \cdot \hat{\tau}_{0}\right),
\end{aligned}
$$

where $\hat{\tau}_{0}=\mathbf{B} / B$, and $m$ and $q$ are the mass and the electric charge of the test particle, respectively. The electric and magnetic fields $\mathbf{E}$ and $\mathbf{B}$ depend in general on the position and time. The trajectory of the guiding center can be obtained, in principle, by solving Eqs. (1)-(3) simultaneously, once the space and time dependence of the electric and magnetic fields are specified. Equation (3) describes conservation of the adiabatic invariant, i.e. $(d / d t) V_{1}^{2} / B=0$. The guiding-center equations, (1) to (3), are obtained from the equation of motion of a charged particle by averaging it over the rapidly varying phase of the gyration. This procedure is justified when $V_{E} \equiv c E / B \& V_{1}, R_{g} \& L$, and $\left|\partial \hat{\tau}_{0} / \partial t\right|<\omega_{g}$, where $V_{E}$ is the electric drift velocity, $\omega_{g}=q B / m c$ denotes the gyrofrequency, $R_{g}=V_{\perp} / \omega_{g}$ is the gyroradius, and $L=|\nabla B / B|^{-1}$ is a characteristic length of the magnetic field inhomogeneity. In deriving Eqs. (1)-(3), an expansion in a parameter $\epsilon \& 1$ is performed. This parameter $\epsilon$ is given by the order of magnitude of the largest of the quantities $R_{g} / L, V_{E} / V_{1}$, or $\left|\partial \hat{\tau}_{0} / \partial t\right| / \omega_{g}$. The first term on the right-hand side of Eq. (1) is of zeroth order, and the remaing terms are of first order in $\epsilon$. The terms on the right-hand side of Eqs. (2) and (3) are all of zeroth order. In tokamaks, the ratios $R_{g} / L, V_{E} / V_{\perp}$, and $\left|\partial \hat{\tau}_{0} / \partial t\right| / \omega_{g}$ are indeed much smaller than one in general, although of different size. The parameter $R_{g} / L$ has a value between $10^{-3}$ and $10^{-4}$ for electrons, and an order of magnitude larger for deuterons. The electric drift for an electric ficld of $10^{2} \mathrm{~V} / \mathrm{cm}$ and a magnetic field $B \sim 10 \mathrm{KG}$ is of the order of $V_{E^{\sim}} \sim 10^{6} \mathrm{~cm} / \mathrm{sec}$, and the 
thermal velocity $v_{\text {th }}$ in a plasma with an edge temperature of $100 \mathrm{eV}$ is about $5 \times 10^{8} \mathrm{~cm} / \mathrm{sec}$ for electrons, and $2 \times 10^{7}$ $\mathrm{cm} / \mathrm{sec}$ for deuterons. Thus for $V_{\perp} \sim v_{\text {th }}$ the ratio $V_{E} / V_{\perp}$ is approximately $2 \times 10^{-3}$ for electrons, and $5 \times 10^{-2}$ for deuterons. The ratio $\left|\partial \hat{\tau}_{0} / \partial t\right| / \omega_{g}$ is about $10^{-8}$ for electrons and $10^{-4}$ for deuterons.

We assume that the magnetic and electric fields are of the form

$$
\begin{aligned}
& \mathbf{B}=\mathbf{B}_{0}(\mathbf{r})+\mathbf{b}(\mathbf{r}, t), \\
& \mathbf{E}=\mathbf{E}_{0}(\mathbf{r})+\mathbf{e}(\mathbf{r}, t),
\end{aligned}
$$

where $\mathbf{b}$ and $\mathbf{e}$ represent the fluctuations in the magnetic and electric fields observed in tokamaks, and analyze the transport induced by these fluctuations. Although the experimental information does not allow general conclusions we will assume that $b<B_{0}$ and $e \lesssim E_{0}$. Further, it has been observed $^{1}$ that the magnitude of the magnetic field fluctuations in the direction perpendicular to the average magnetic field $\mathbf{B}_{0}$, i.e., the magnitude of $b_{1}$, at plasma edge is about $10^{-4}$ times the magnitude of the total magnetic field for small tokamaks and even smaller for large tokamaks. This magnitude seems to increase in going from the edge to the central plasma regions. The magnitude of the magnetic field fluctuations in the parallel direction, i.e., $b_{\|}$, is not well known, but it is always found to be lower than the magnitude of the fluctuations in the perpendicular direction $^{1}$ [in the Tokamak Chauffage Alfvén (TCA), ${ }^{17}$ this factor is $1 / 3$, and in the MACROTOR Tokamak ${ }^{18}$ it is lower than 1/10]. In order to perform an expansion of the guiding-center equations, based on these quantities, we introduce the parameters

$$
\delta_{1} \equiv b_{1} / B \sim 10^{-4}
$$

and

$$
\delta_{\|} \equiv b_{\|} / B<\delta_{\perp} .
$$

Similarly, we also introduce two additional parameters $\alpha_{\perp} \equiv e_{1} / E_{0}$ and $\alpha_{\|} \equiv e_{\|} / E_{0}$ to characterize the magnitude of the fluctuations of the electric field in the perpendicular and parallel directions relative to $E_{0}$, which is an average electric field. However, to estimate the order of magnitude of these parameters we first have to know $E_{0}$. The reported measurements of the radial electrical fields in tokamaks show that the electric potential varies significantly depending on the plasma conditions. In the plasmas of the Impurity Study Experiment B (ISX-B) ${ }^{19}$ and TM- ${ }^{20}$ the electric potential is negative at the plasma center with respect to the plasma edge, and increases with distance from the center, acquiring positive values near the edge. The corresponding maximum radial electric field is approximately $10^{2} \mathrm{~V} / \mathrm{cm}$. In the Rensselar Torus (RENTOR) ${ }^{21}$ a positive electric potential is observed at the plasma center. Measurements of the electric potential fluctuations, $\widetilde{\phi}(\mathbf{r}, t)$, in the ISX-B, ${ }^{22}$ the California Institute of Technology Tokamak (CALTECH) ${ }^{23}$ and the MACROTOR ${ }^{24}$ [see Ref. (1)], show that the magnitude of the fluctuations increase in general going from the plasma edge to the central regions. The maximal variations of the electric potential are up to $30 \mathrm{~V}$. On the other hand, the wavelength spectra of the potential fluctuations perpendicular to the magnetic field show a maximum at about $1 \mathrm{~cm}$. Hence, we can estimate an upper limit for the magnitude of the electric field fluctuations $e_{\perp}$ as $30 \mathrm{~V} / \mathrm{cm}$ (for the CALTECH Tokamak, values of $10 \mathrm{~V} / \mathrm{cm}$ are reported ${ }^{23}$ ). The magnitude of the electric field fluctuations along the magnetic field lines is not well known, but since the wavelength of the potential fluctuations along $\mathbf{B}_{0}$ are much greater than the wavelength perpendicular to $B_{0}$ (at least a factor 6 in the MACROTOR Tokamak ${ }^{24}$ ), we may conclude that $e_{\|}<e_{1}$. In summary, we find the order of magnitude of the parameters $\alpha_{1}$ and $\alpha_{\|}$as

$$
\begin{aligned}
& \alpha_{1}=e_{\perp} / E_{0} \sim 0.1-1, \\
& \alpha_{\|}=e_{\|} / E_{0}<\alpha_{\perp} .
\end{aligned}
$$

The magnitude of the drifts in Eq. (1) arising from the spatial gradients of the fluctuating magnetic fields can be estimated by their characteristic lengths, which may be taken as their wavelength $\lambda_{\|}$and $\lambda_{1}$ in the parallel and perpendicular directions. Typically $\lambda_{1} \sim 0.05-2 \mathrm{~cm}$ and $\lambda_{\|}>\lambda_{1}\left[\lambda_{\|} \sim 200 \lambda_{1}\right.$ in the Texas Experimental Tokamak (TEXT) $)^{3}$. We can define the parameters

$$
\begin{aligned}
& \gamma_{\perp} \equiv \lambda_{1} / L \sim 10^{-2}-10^{-4}, \\
& \gamma_{\|} \equiv \lambda_{\|} / L>\gamma_{\perp},
\end{aligned}
$$

to express $\lambda_{\perp}$ and $\lambda_{\|}$relative to the characteristic length of the tokamak.

Another parameter we need to measure is the magnitude of the drift caused by the time variations in $\hat{\tau}_{0}$. This is defined by $\mu \equiv\left(2 \pi f / \omega_{g}\right) \delta_{1}$, where $f$ is the frequency of the magnetic field fluctuations, which ranges up to $1 \mathrm{MHz}$. Since the gyrofrequency $\left(\omega_{g} / 2 \pi\right)$ is about $10^{10} \sec ^{-1}$ for electrons, and $10^{6} \mathrm{sec}^{-1}$ for ions we find that

$$
\mu \lesssim \begin{cases}10^{-8}, & \text { for electrons, } \\ 10^{-4}, & \text { for deuterons. }\end{cases}
$$

We are now in a position to expand the right-hand sides of Eqs. (1) - (3) using Eqs. (4) and the parameters introduced in Eqs. (5)-(8) and keep only the most important terms. The unit vector $\hat{\tau}_{0}$ in Eq. (1) can be expanded as

$$
\hat{\tau}_{0}=\frac{\mathbf{B}_{0}}{B}+\frac{\mathbf{b}_{1}}{B_{0}}+O\left(\delta_{1}^{2}\right),
$$

where we have used Eqs. (5) and ignored $b_{\|} / B_{0}$. The electric drift in Eq. (1), which is of the order of $V_{E} / V_{1}$ relative to the term $V_{\|} \hat{\tau}_{0}$, can be approximated as

$$
c\left(\mathbf{E} \times \hat{\tau}_{0} / B\right)=\frac{c}{B_{0}^{2}}\left(\mathbf{E}_{0}+\mathbf{e}\right) \times\left(\mathbf{B}_{0}+\mathbf{b}\right)+O\left(V_{E} \delta_{\|}\right),
$$

where the $\left(\mathbf{e} \times \mathbf{B}_{0}\right)$ term is of the order $\alpha_{1}$ compared to the $\left(\mathbf{E}_{0} \times \mathbf{B}_{0}\right)$ term, and must be retained because $\alpha_{1} \sim 0.1-1$ [cf. Eq. (6)]. The $\left(\mathbf{E}_{0}+\mathbf{e}\right) \times \mathbf{b}$ term is of order $\delta_{1}$ and is also retained.

The $\nabla B$-drift term in Eq. (1) is of the order of $R_{g} / L$ compared to the term $V_{\|} \widehat{\tau}_{0}$, and can be simplified as 


$$
\begin{aligned}
\frac{m c}{2 q B} V_{1}^{2}\left(\frac{\hat{\tau}_{0} \times \nabla B}{B}\right)= & \frac{m c}{2 q B_{0}^{3}} V_{1}^{2}\left[\mathbf{B}_{0} \times \nabla B_{0}+\mathbf{B}_{0} \times \nabla\left(\frac{\mathbf{B}_{0} \cdot \mathbf{b}}{B_{0}}\right)\right. \\
& \left.+\mathbf{b} \times \nabla B_{0}+O\left(B_{0}^{2} \delta_{\|} / L\right)\right]
\end{aligned}
$$

The second term in this equation is of the order of $\delta_{\|}\left(L / \lambda_{\perp}\right)=\delta_{\|} / \gamma_{\perp}$ as compared to the first term, and must be retained because the ratio $\delta_{\|} / \gamma_{1}$ can be close to unity [see Eqs. (5) and (7)], although it is always less than one. The third term is of the order $\delta_{1}$ as compared to the first term, and it also is retained.

The curvature drift in Eq. (1) is the order of $R_{g} / L$ with respect to the term $V_{\|} \hat{\tau}_{0}$, and can be approximated by

$$
\begin{aligned}
& \frac{m c}{q B} V_{\|}^{2}\left\{\hat{\tau}_{0} \times\left[\left(\hat{\tau}_{0} \cdot \nabla\right) \hat{\tau}_{0}\right]\right\} \\
&=\frac{m c V_{\|}^{2}}{q B_{0}^{4}}\left\{\mathbf{B}_{0} \times\left[\left(\mathbf{B}_{0} \cdot \nabla\right) \mathbf{B}_{0}\right]+\mathbf{B}_{0} \times\left[\left(\mathbf{B}_{0} \cdot \nabla\right) \mathbf{b}\right]+\mathbf{B}_{0}\right. \\
&\left.\times\left[(\mathbf{b} \cdot \nabla) \mathbf{B}_{0}\right]+\mathbf{b} \times\left[\left(\mathbf{B}_{0} \cdot \nabla\right) \mathbf{B}_{0}\right]+O\left(B_{0}^{3} \delta_{1}^{2} / \lambda_{1}\right)\right\}
\end{aligned}
$$

The second term on the right-hand side is of the order of $\delta_{\perp}\left(L / \lambda_{\|}\right)=\delta_{1} / \gamma_{\|}$in comparison with the first term. Since $\gamma_{\|}$can be lower than one [see Eq. (5)] we must retain this term. The third and fourth terms are of the order $\delta_{1}$ and are also kept in our equations.

In the expressions (11) and (12) the expansion is made up to the leading terms in the fluctuations. Although these terms may be small compared with the nonfluctuating terms, they are retained since they are the leading terms when $\mathbf{B}_{0}$ is uniform.

Finally, the last term on the right-hand side of Eq. (1), i.e., $\partial \hat{\tau}_{0} / \partial t$ is of the order $\mu$ as compared to $V_{\|} \hat{\tau}_{0}$, and can thus be neglected [see Eq. (8)].

We conclude from the above estimates that Eq. (1) can be approximated by

$$
\begin{aligned}
\frac{d \mathbf{R}}{d t}= & V_{\|}\left(\frac{\mathbf{B}_{0}+\mathbf{b}_{1}}{B_{0}}\right)+\frac{c}{B_{0}^{2}}\left(\mathbf{E}_{0}+\mathbf{e}\right) \times\left(\mathbf{B}_{0}+\mathbf{b}\right) \\
& +\frac{m V_{1}^{2} c}{2 q B_{0}^{3}}\left\{\mathbf{B}_{0} \times \nabla\left[B_{0}+b_{\|}\right]+\mathbf{b} \times \nabla B_{0}\right\} \\
& +\frac{m V_{\| c}^{2}}{q B_{0}^{4}}\left\{\mathbf{B}_{0} \times\left[\left(\mathbf{B}_{0} \cdot \nabla\right)\left(\mathbf{B}_{0}+\mathbf{b}\right)+(\mathbf{b} \cdot \nabla) \mathbf{B}_{0}\right]\right. \\
& \left.+\mathbf{b} \times\left[\left(\mathbf{B}_{0} \cdot \nabla\right) \mathbf{B}_{0}\right]\right\},
\end{aligned}
$$

when studying the diffusion of the guiding centers induced by random tokamak-like fluctuations.

We now turn to Eq. (2), and consider the term

$$
(q / m) \mathbf{E} \cdot \hat{\tau}_{0}=\left(q / m B_{0}\right)\left(\mathbf{B}_{0} \cdot \mathbf{E}_{0}+\mathbf{B}_{0} \cdot \mathbf{e}+\mathbf{b} \cdot \mathbf{E}_{0}+\mathbf{b} \cdot \mathbf{e}\right)
$$

As compared to the first term $\mathbf{B}_{0} \cdot \mathbf{F}_{0}$, the second, third, and fourth terms are of the order of $\alpha_{\|}, \delta_{1}$, and $\delta_{1} \alpha_{1}$, respectively. Hence, the last two terms can be neglected, so that

$$
(q / m) \mathbf{E} \cdot \hat{\tau}_{0}=\left(q / m B_{0}\right)\left(\mathbf{B}_{0} \cdot \mathbf{E}_{0}+\mathbf{B}_{0} \cdot \mathbf{e}\right) .
$$

The term $\nabla \cdot \hat{\tau}_{0}$ in Eqs. (2) and (3) can be approximated as

$$
\boldsymbol{\nabla} \cdot \hat{\tau_{0}}=-\mathbf{B} / B^{2} \cdot \boldsymbol{\nabla} B=-\left(1 / B_{0}^{2}\right)\left[\left(\mathbf{B}_{0}+\mathbf{b}_{\perp}\right) \cdot \nabla B_{0}\right],
$$

where the terms of the order of $\left(\delta_{\|} / \lambda_{\|}\right),\left(\delta_{1}^{2} / \lambda_{1}\right)$, and higher order have been neglected. Hence, we can write Eqs. (2) and (3) as

$$
\begin{aligned}
& \frac{d V_{\|}}{d t}=\frac{q}{m B_{0}} \mathbf{B}_{0} \cdot\left(\mathbf{E}_{0}+\mathbf{e}\right)-\frac{V_{1}^{2}}{2 B_{0}^{2}}\left[\left(\mathbf{B}_{0}+\mathbf{b}_{1}\right) \cdot \nabla B_{0}\right], \\
& \frac{d V_{\perp}}{d t}=\frac{V_{1} V_{0}}{2 B_{0}^{2}}\left[\left(\mathbf{B}_{0}+\mathbf{b}_{1}\right) \cdot \nabla B_{0}\right] .
\end{aligned}
$$

In conclusion, the motion of the guiding center can be studied in tokamaks using the coupled set of equations (13), (17), and (18), when the deterministic magnetic and electric fields $\mathbf{B}_{0}(\mathbf{r})$ and $\mathbf{E}_{0}(\mathbf{r})$ are given, and the statistical properties of the fluctuating fields $\mathbf{b}(\mathbf{r}, t)$ and $\mathbf{e}(\mathbf{r}, t)$ are specified. Here it must be kept in mind that $b_{\|} \leqslant b_{1}$ is assumed, so that the case $b_{\perp}=0$ and $b_{\| \neq} \neq 0$ cannot be treated with the set of equations we have derived. It is clear that the fields in these equations are to be evaluated at the instantaneous position $\mathbf{R}(t)$ of the guiding center. In order to proceed analytically with the study of the effect of fluctuations in the local fields on the motion of the guiding center in a more quantitative manner, we are forced to introduce futher simplifications. Since the correlation lengths of the fiuctuations are much smaller than the characteristic plasma dimensions (unfortunately not always true for the parallel correlations), the diffusion induced by fluctuations can be considered as a local phenomenon; this enables us to take the deterministic part of the ficlds as a constant and the statistical properties of the fluctuations as cylindrically symmetric. The effects of the toroidal geometry can, however, still be present through the correlation functions.

\section{THE MODEL: UNIFORM $B_{0}$ AND $E_{0}$}

Let us assume the average magnetic and electric fields are uniform and in the $z$ direction, i.e., $\mathbf{B}_{0}=B_{0} \hat{z}$ and $\mathbf{E}_{0}=E_{0} \hat{z}$, where $\hat{z}$ is the unit vector, and introduce the effect of collisions in the parallel motion through a random collision term $\left[-v_{c} V_{\|}+F(t)\right]$ in the right-hand side of Eq. (17). Here $v_{c}$ is a collision frequency and $F(t)$ is a random Langevin force, which does not correlate with e or $\mathbf{b}$. This Brownian diffusion model has been used before ${ }^{25}$ to study the diffusion of the guiding center of a test particle in a fluctuating magnetic field.

With the above idealizations, the guiding-center equations (13), (17), and (18) are modified as follows:

$$
\begin{aligned}
\frac{d \mathbf{R}(t)}{d t}= & V_{\|}\left(\hat{z}+\frac{\mathbf{b}_{1}}{B_{0}}\right)+c \frac{\mathbf{e} \times \mathbf{B}_{0}}{B_{0}}+\frac{m V_{1}^{2} c}{2 q B_{0}^{3}} \mathbf{B}_{0} \times \nabla b_{\|} \\
& +\frac{m V_{\|}^{2} c}{q B_{0}^{4}} \mathbf{B}_{0} \times\left(\mathbf{B}_{0} \cdot \nabla\right) \mathbf{b}, \\
\frac{d V_{\|}(t)}{d t}= & \frac{q}{m}\left(E_{0}+e_{z}\right)-v_{c} V_{\|}+F,
\end{aligned}
$$




$$
\frac{d V_{1}}{d t}=0 .
$$

Equations (19) and (20) represent a set of two coupled stochastic differential equations for $\mathbf{R}(t)$ and $V_{\|}(t)$ in which $\mathbf{b}, \mathbf{e}$, and $F$ are the input random processes whose statistical properties are to be modeled. Equation (21) shows the conservation of the adiabatic invariant for a constant magnetic field. In Cartesian components Eqs. (19) and (20) read

$$
\begin{aligned}
& \frac{d X(t)}{d t}=V_{\|} \frac{b_{x}}{B_{0}}+c \frac{e_{y}}{B_{0}}-\frac{V_{1}^{2}}{\omega_{g} B_{0}} \frac{\partial b_{z}}{\partial Y}-\frac{V_{\|}^{2}}{\omega_{g} B_{0}} \frac{\partial b_{y}}{\partial Z}, \\
& \frac{d Y(t)}{d t}=V_{\|} \frac{b_{y}}{B_{0}}-c \frac{e_{x}}{B_{0}}+\frac{V_{\perp}^{2}}{\omega_{g} B_{0}} \frac{\partial b_{z}}{\partial X}+\frac{V_{\|}^{2}}{\omega_{g} B_{0}} \frac{\partial b_{x}}{\partial Z}, \\
& \frac{d Z(t)}{d t}=V_{\|}, \\
& \frac{d V_{\|}(t)}{d t}=-v_{c} V_{\|}+\frac{q}{m}\left(E_{0}+e_{z}\right)+F .
\end{aligned}
$$

In general $\mathbf{b}$ and $\mathbf{e}$ might exhibit time and space dependence. The case including space dependence is more complex and will be treated in Sec. V by using an iterative procedure based on Novikov's theorem presented in Sec. IV. However, the case in which $\mathbf{b}$ and e show only time dependence can be solved exactly and is presented in the following section. The results obtained there are also used later to analyze the accuracy of this approach.

\section{DIFFUSION DUE TO STOCHASTIC FIELDS WITHOUT SPACE DEPENDENCE}

In this section we consider $\mathbf{b}=\mathbf{b}(t)$ and $\mathbf{e}=\mathbf{e}(t)$ and evaluate $\left\langle V_{\|}\right\rangle,\left\langle V_{\|}^{2}\right\rangle,\langle X\rangle$, and $\left\langle X^{2}\right\rangle$ through Eqs. (22) to (25) under the assumption that $\mathbf{b}$ and $\mathbf{e}$ are stationary random fluctuations with zero mean, and are uncorrelated (although this seems to be a strong assumption, there is, however, some experimental evidence supporting it for at least low-frequency fluctuations). ${ }^{1,3}$ Here $\left\langle V_{\|}\right\rangle$means the ensemble average of $V_{\|}$. Purely time-dependent electric and magnetic fields are, of course, inconsistent with Maxwell's equations. However, in analytical treatments of the transport induced by stochastic fluctuations this is not an uncommon assumption because the conclusions are useful for understanding the basic physics involved in the process. The results we derive here are used later in Sec. VI to analyze the structure of the diffusion coefficients for the case when both time and space dependences are included.

Following the procedure presented in Appendix A we obtain from Eq. (25) that

$$
\left\langle V_{\|}(t)\right\rangle=V_{0} e^{-v_{c} t}+\left(q E_{0} / m v_{c}\right)\left(1-e^{-v_{c} t}\right)
$$

and

$$
\left\langle\delta V_{\|}^{2}(t)\right\rangle=v_{c}^{-1} e^{-v_{c} t} \int_{0}^{t} d u R_{s}(|u|)\left(e^{v_{c}(t-u)}-e^{-v_{c}(t-u)}\right)
$$

where $\delta V_{\|}=V_{\|}-\left\langle V_{\|}\right\rangle, R_{s}$ is the correlation of the fluctuating component of the right-hand side (rhs) of Eq. (25) and $V_{0}$ is a constant initial velocity for all ensemble realizations. Expression (27) describes stochastic heating due to electric fluctuations and diffusion in velocity space (see Appendix A). From Eq. (22) we can evaluate $\langle\delta X(t)\rangle$ and $\left\langle\delta X^{2}(t)\right\rangle$. The diffusion coefficient perpendicular to the average magnetic field, $D_{1}(t)=\frac{1}{2} d\left\langle\delta^{2} X(t)\right\rangle / d t$, is given by

$$
\begin{aligned}
D_{1}(t)= & \left\langle V_{\|}(t)\right\rangle \int_{0}^{t} d \tau\left\langle V_{\|}(t-\tau)\right\rangle\left\langle b_{x}(t) b_{x}(t-\tau)\right\rangle / B_{0}^{2} \\
& +\int_{0}^{t} d \tau\left\langle\delta V_{\|}(t) \delta V_{\|}(t-\tau)\right\rangle\left\langle b_{x}(t) b_{x}(t-\tau)\right\rangle / B_{0}^{2} \\
& +c^{2} \int_{0}^{t} d \tau\left\langle e_{y}(t) e_{y}(t-\tau)\right\rangle / B_{0}^{2}
\end{aligned}
$$

The last term, which does not depend either on electric charge or the mass of the particle, describes the contribution to diffusion of the stochastic electric drift. It is believed to be one of the main mechanisms leading to anomalous transport. ${ }^{1}$ The first and second terms give the diffusion resulting from the motion of the guiding center along stochastic field lines. These contributions have been the subject of multiple papers [see Ref. (9)]. When the parallel correlation length is much smaller than the torus parallel dimensions (as in our case), it does not seem to be relevant for explaining anomalous transport since $|\mathrm{b}| / B_{0}$ is very small $^{2}$ (if quasilinear formulas apply). The first term appears due to a nonvanishing average parallel motion, which for $t \gg v_{c}^{-1}$, is given by $\left\langle V_{\|}\right\rangle=q E_{0} / m v_{c}$. This term gives the diffusion coefficient associated to the loop voltage in a tokamak. For small times, $t \ll v_{c}^{-1}$, this diffusion coefficient increases linearly in time.

To estimate the size of the terms involved in Eq. (28), let us introduce the steady-state perpendicular decorrelation time of the magnetic and electric fluctuations as

$$
\tau_{\perp b}=\frac{1}{\left\langle b_{x}(0) b_{x}(0)\right\rangle} \int_{0}^{\infty} d \tau\left\langle b_{x}(0) b_{x}(\tau)\right\rangle
$$

and

$$
\tau_{\perp e}=\frac{1}{\left\langle e_{y}(0) e_{y}(0)\right\rangle} \int_{0}^{\infty} d \tau\left\langle e_{y}(0) e_{y}(\tau)\right\rangle
$$

Thus, for $t \rightarrow \infty$ Eq. (28) goes into (see Appendix A)

$$
\begin{aligned}
D_{\perp}= & \left\langle V_{\|}\right\rangle^{2} \frac{\left\langle b_{x}(0) b_{x}(0)\right\rangle}{B_{0}^{2}} \tau_{\perp b} \\
& +\frac{1}{B_{0}^{2}} \int_{0}^{\infty} d \tau R_{V_{\|}}(\tau)\left\langle b_{x}(0) b_{x}(\tau)\right\rangle \\
& +\frac{c^{2}}{B_{0}^{2}}\left\langle e_{y}(0) e_{y}(0)\right\rangle \tau_{\perp e},
\end{aligned}
$$

where $R_{V_{\|}}(\tau)=\lim _{t \rightarrow \infty}\left\langle\delta V_{\|}(t) \delta V_{\|}(t-\tau)\right\rangle$.

In the case $\tau_{1 b}$ is much smaller than the decorrelation time of $\delta_{V_{\|}}$, we can approximate the second term in the rhs of Eq. (30) by 


$$
v_{\text {th }}^{2} \frac{\left\langle b_{x}(0) b_{x}(0)\right\rangle}{B_{0}^{2}} \tau_{b 1}
$$

with $v_{\text {th }}^{2}=R_{v_{\|}}(0)$, the square of the thermal velocity in parallel direction. The first term on the rhs of Eq. (30) becomes comparable or greater than the second one only when $\left\langle V_{\|}\right\rangle \gtrsim v_{\text {th }}$. This may be the case when tangential neutral beam injection is present in tokamaks, or in the case of run-away electrons.

Let us now evaluate $D_{1}$ for the CALTECH tokamak. ${ }^{23}$ In this case $\tau_{\perp e} \sim 2 \times 10^{-5} \mathrm{sec},|\mathrm{e}| \sim 10 \mathrm{~V} / \mathrm{cm}$ which, together with $B_{0}=3.5 \mathrm{KG}$, gives $c e_{y} / B_{0} \sim 3.3 \times 10^{5} \mathrm{~cm} / \mathrm{sec}$. The diffusion coefficient due to stochastic electric drifts is then

$$
D_{\text {L,ELEC }} \sim 2 \times 10^{6} \mathrm{~cm}^{2} / \mathrm{sec},
$$

which is an extremely high value in comparison with the experimently observed anomalous diffusion coefficients $D \sim 10^{2}-10^{3} \mathrm{~cm}^{2} / \mathrm{sec}$.

The diffusion resulting from the magnetic fluctuations can be evaluated by taking $\tau_{1 b} \sim \tau_{1 e}$ and $b_{x} / B_{0} \sim 10^{-4}$ together with $v_{\text {th, },} \sim 2 \times 10^{8} \mathrm{~cm} / \mathrm{sec}$ and $v_{\mathrm{th}, i} \sim 8 \times 10^{6} \mathrm{~cm} /$ sec. This yields

$$
D_{1, \mathrm{MAG}}= \begin{cases}8 \times 10^{3} \mathrm{~cm}^{2} / \mathrm{sec}, & \text { for electrons, } \\ 13 \mathrm{~cm}^{2} / \mathrm{sec}, & \text { for ions },\end{cases}
$$

which is lower than the electric diffusion coefficient, Eq. (32), but still of importance for electrons. Notice that electrons diffuse faster than ions, thus the diffusion mechanism provided by the magnetic fluctuations via weak destruction of magnetic surfaces should tend to develop a positive ambipolar electric field.

\section{ITERATIVE TECHNIQUE BASED ON NOVIKOV'S FORMULA}

Let us consider a system whose behavior is described by a stochastic differential equation of the form

$$
\frac{d \mathbf{r}}{d t}=\mathbf{f}[\mathbf{r}(t), t ; \tilde{\omega}],
$$

where $\mathbf{r}(t)$ is a state vector of arbitrary dimension and the function $\mathbf{f}(\mathbf{r}, t ; \tilde{\omega})$ represents a vector function of $\mathbf{r}, t$ for each realization of a random variable $\widetilde{\omega} .^{26}$ We now introduce the density $\rho(\mathbf{r}, t ; \widetilde{\omega})=\delta[\mathbf{x}-\mathbf{r}(t ; \widetilde{\omega})]$ for each realization, so that its average over realizations

$$
P(\mathbf{x}, t)=\int d \tilde{\omega} D(\widetilde{\omega}) \rho(\mathbf{x}, t ; \tilde{\omega})=\langle\rho(\mathbf{x}, t)\rangle
$$

is the probability density function. From this function we can obtain the probability that at a given time $t$, and for a particular realization, the value of $\mathbf{r}$ lies in $d \mathbf{x}$ around $\mathbf{x}$.
The density function $\rho(x, t ; \widetilde{\omega})$ satisfies the following continuity equation:

$$
\frac{\partial}{\partial t} \rho(\mathbf{x}, t ; \widetilde{\omega})=-\nabla \cdot \mathbf{f}(\mathbf{x}, t ; \tilde{\omega}) \rho(\mathbf{x}, t ; \tilde{\omega})=\mathscr{L}(t ; \widetilde{\omega}) \rho(\mathbf{x}, t ; \widetilde{\omega}),
$$

where $\mathscr{f}^{\prime}(\tilde{\omega})$ is the stochastic Liouville operator. ${ }^{27}$

The formal solution of Eq. (36) is

$$
\rho(\mathbf{x}, t ; \widetilde{\omega})=\exp _{t}\left(\int_{0}^{t} d s \mathscr{L}(s ; \tilde{\omega})\right) \rho(\mathbf{x}, 0 ; \tilde{\omega}),
$$

where the operator on the right-hand side denotes timeordered exponential, ${ }^{28}$ defined by

$$
\begin{aligned}
& \exp _{t}\left(\int_{0}^{t} d s \mathscr{L}(s ; \widetilde{\omega})\right) \\
&=1+\sum_{n=1}^{\infty} \int_{0}^{t} d t_{1} \int_{0}^{t_{1}} d t_{2} \cdots \int_{0}^{t_{n-1}} d t_{n} \\
& \times \mathscr{L}\left(t_{1} ; \tilde{\omega}\right) \cdots \mathscr{L}\left(t_{n} ; \tilde{\omega}\right) .
\end{aligned}
$$

Averaging Eq. (37) over realizations and assuming that the initial value $\rho(\mathbf{x}, 0 ; \widetilde{\omega})$ is not correlated with the realizations, i.e., is independent of $\widetilde{\omega}$, one formally obtains

$$
P(\mathbf{x}, t)=\left\langle\exp _{t} \int_{0}^{t} d s \mathscr{L}(s)\right\rangle P(\mathbf{x}, 0) .
$$

Our purpose is to obtain an equation for $P(x, t)$ using a theorem due to Novikov ${ }^{29}$ for the case when $\mathbf{f}(\mathbf{x}, t ; \widetilde{\omega})$ is a Gaussian stochastic process with zero mean. Here Gaussian process means that the probability of finding a given value of $\mathbf{f}$ at $(\mathbf{x}, t)$ is a Gaussian distribution. ${ }^{30}$

Before considering the general case, we first introduce this technique in the one-dimensional case of Eq. (34) and assume that $f$ is space independent.

\section{A. A simple one-dimensional case}

Let us consider the stochastic equation

$$
\frac{d x}{d t}=f(t ; \tilde{\omega})
$$

and the stochastic Liouville operator

$$
\mathscr{L}(t ; \tilde{\omega})=-f(t ; \tilde{\omega}) \frac{\partial}{\partial x} .
$$

The time ordering in Eq. (37) is now not needed since $\mathscr{L}(t, \widetilde{\omega})$ and $\mathscr{Z}\left(t^{\prime} ; \widetilde{\omega}\right)$ commute.

Assuming that $f(t ; \tilde{a})$ is a stationary Gaussian process with zero mean, and autocorrelation function

$$
R(\tau)=\langle f(t) f(t+\tau)\rangle,
$$

one obtains from Eq. (37)

$$
P(x, t)=\exp \left(\int_{0}^{t} d s(t-s) R(s)\right) P(x, 0),
$$

which satisfies the following Fokker-Planck equation 


$$
\frac{\partial P(x, t)}{\partial t}=\left(\int_{0}^{t} d s R(s)\right) \frac{\partial^{2} P(x, t)}{\partial x^{2}} .
$$

We shall use this exact result to compare with the approximate results of the iterative technique based on Novikov's theorem that we describe right below.

The continuity equation satisfied by $\rho(x, t ; \widetilde{\omega})$ in the case of Eq. (40) is

$$
\frac{\partial \rho}{\partial t}=-f(t ; \tilde{\omega}) \frac{\partial}{\partial x} \rho(x, t ; \tilde{\omega}) .
$$

By taking the ensemble average of both sides of this equation we obtain

$$
\frac{\partial}{\partial t} P(x, t)=-\frac{\partial}{\partial x}\langle f(t) \rho(x, t)\rangle .
$$

Novikov's theorem ${ }^{29}$ establishes that the correlation of a Gaussian, zero mean stochastic process with a functional that depends on it, is given by

$$
\langle f(t) \rho(x, t)\rangle=\int_{0}^{\infty} d s\langle f(t) f(s)\rangle\left\langle\frac{\delta \rho[x, t ; f]}{\delta f(s) d s}\right\rangle,
$$

where the functional derivative in the right-hand side is defined as ${ }^{31}$

$$
\frac{\delta \rho[x, t ; f]}{\delta f(s) d s}=\left.\frac{d}{d \epsilon} \rho[x, t ; f(t)+\epsilon \delta(t-s)]\right|_{\epsilon=0} .
$$

The solution of Eq. (45) can be constructed as

$$
\rho[x, t ; f]=\delta\left(x-x_{0}-\int_{0}^{t} d s f(s)\right),
$$

from which

$$
\frac{\delta \rho[x, t ; f]}{\delta f(s) d s}=-U(t-s) \frac{\partial}{\partial x} \rho(x, t),
$$

where $U(t)$ is the step function.

If we substitute Eq. (50) together with (48) in Eq. (46) we reproduce the exact Fokker-Planck equation in Eq. (44).

In the phase-space dependent case the solution of the continuity equation is, in general, not available in closed form; it then becomes necessary to resort to an iterative method. For this, we integrate formally Eq. (45) yielding

$$
\rho(x, t)=\rho(x, 0)-\frac{\partial}{\partial x} \int_{0}^{t} d s f(s) \rho(x, s)
$$

from which

$$
\begin{aligned}
\left\langle\frac{\delta \rho[x, t ; f]}{\delta f(s) d s}\right\rangle= & -\frac{\partial}{\partial x}(P(x, s) U(t-s) \\
& \left.+\int_{0}^{t} d u\left\langle f(u) \frac{\delta \rho[x, u ; f]}{\delta f(s) d s}\right\rangle\right) .
\end{aligned}
$$

Substituting Eq. (52) in Eq. (46) together with Eq. (47) we obtain

$$
\begin{aligned}
\frac{\partial P(x, t)}{\partial t}= & \int_{0}^{t} d s R(t-s) \frac{\partial^{2}}{\partial x^{2}} P(x, s)+\frac{\partial^{2}}{\partial x^{2}} \int_{0}^{\infty} d s R(t-s) \\
& \times \int_{0}^{t} d u\left\langle f(u) \frac{\delta \rho[x, u ; f]}{\delta f(s) d s}\right\rangle
\end{aligned}
$$

This is still an exact equation. In fact, if one calculates the functional derivative on the right-hand side using the exact formula in Eq. (49), one reproduces again the exact Fokker-Planck equation (44). However, one can generate an expansion from Eq. (53) by repeated application of Novikov's formula, given in Eq. (47). The result after the second iteration is

$$
\begin{aligned}
\frac{\partial P(x, t)}{\partial t}= & \int_{0}^{t} d s R(t-s) \frac{\partial^{2}}{\partial x^{2}} P(x, s)+\int_{0}^{t} d s R(t-s) \\
& \times \int_{s}^{t} d u \int_{0}^{u} d v R(u-v) \frac{\partial^{4}}{\partial x^{4}}[U(s-v) P(x, v) \\
& +U(v-s) P(x, s)]+\frac{\partial^{6}}{\partial x^{6}}[\cdots] .
\end{aligned}
$$

Since this is the procedure we follow in the phase-space dependent case, it is convenient to check how well Eq. (54) reproduces the moments

$$
\overline{x^{n}}(t)=\int d x x^{n} P(x, t) .
$$

We first observe that Eq. (54) reproduces the exact Fokker-Planck equation (44) when $f(t)$ is delta correlated. Second, it yields the first four moments exactly. Indeed, one finds from Eq. (54), that $\bar{x}(t)=\overline{x^{3}}(t)=0$ and

$$
\frac{d}{d t} \overline{x^{2}}(t)=2 \int_{0}^{t} d s R(s)
$$

and

$$
\frac{d}{d t} \overline{x^{4}}(t)=24 \int_{0}^{t} d s R(s) \int_{0}^{t} d u(t-u) R(u),
$$

which also follow from Eq. (44). Clearly, the odd order moments always vanish and the number of even order moments that can be calculated correctly from Eq. (54) is determined by the highest even-order derivatives retained in the truncation.

\section{B. The case with space- and time-dependent fluctuations}

Here we generalize the procedure described above to study the case of a system whose behavior is governed by the stochastic differential equation (34) with $\mathbf{f}(\mathbf{r}, t)$ a vector Gaussian process with zero mean. In order to implement the procedure we average Eq. (36) over realizations to obtain

$$
\frac{\partial}{\partial t} P(\mathbf{x}, t)=-\frac{\partial}{\partial x_{j}}\left\langle f_{j}(\mathbf{x}, t) \rho(\mathbf{x}, t)\right\rangle,
$$

where summation over repeated indices is understood; we now use Novikov's formula 


$$
\begin{aligned}
\left\langle f_{j}(\mathbf{x}, t) \rho(\mathbf{x}, t)\right\rangle= & \int \mathbf{d} x^{\prime} \int_{0}^{\infty} d s R_{j k}\left(\mathbf{x}, t ; \mathbf{x}^{\prime}, s\right) \\
& \times\left\langle\frac{\delta \rho[\mathbf{x}, t ; \mathbf{f}]}{\delta f_{k}\left(\mathbf{x}^{\prime}, s\right) d \mathbf{x}^{\prime} d s}\right\rangle
\end{aligned}
$$

where

$$
\begin{aligned}
\frac{\delta \rho[\mathbf{x}, t ; \mathbf{f}]}{\delta f_{l}\left(\mathbf{x}^{\prime}, s\right) d \mathbf{x}^{\prime} d s}= & \frac{\partial}{\partial \epsilon} \rho\left[\mathbf{x}, t ; f_{i}(\mathbf{x}, t)\right. \\
& \left.+\epsilon \delta_{i} \delta\left(\mathbf{x}-\mathbf{x}^{\prime}\right) \delta(t-s)\right]\left.\right|_{\epsilon=0}
\end{aligned}
$$

is the functional derivative of first order and

$$
R_{j k}\left(\mathbf{x}, t ; \mathbf{x}^{\prime}, s\right) \equiv\left\langle f_{j}(\mathbf{x}, t) f_{k}\left(\mathbf{x}^{\prime}, s\right)\right\rangle
$$

denotes the correlation functions of the $j$ th and $k$ th components of $\mathbf{f}$.

Formally integrating Eq. (36) we have

$$
\rho(\mathbf{x}, t)=\rho(\mathbf{x}, 0)-\frac{\partial}{\partial x_{m}} \int_{0}^{t} d s f_{m}(\mathbf{x}, s) \rho(\mathbf{x}, s) ;
$$

from which we obtain

$$
\begin{aligned}
\frac{\delta \rho[\mathbf{x}, t ; f]}{\delta f_{k}\left(\mathbf{x}^{\prime}, s\right) d \mathbf{x}^{\prime} d s}= & -\frac{\partial}{\partial x_{l}} \int_{0}^{t} d u \delta_{l k} \delta\left(\mathbf{x}-\mathbf{x}^{\prime}\right) \delta(u-s) \\
& \times \rho(\mathbf{x}, u)-\frac{\partial}{\partial x_{m}} \int_{0}^{t} d u f_{m}(\mathbf{x}, u) \\
& \times \frac{\delta \rho[\mathbf{x}, u ; \mathbf{f}]}{\delta f_{k}\left(\mathbf{x}^{\prime}, s\right) d \mathbf{x}^{\prime} d s}
\end{aligned}
$$

After taking ensemble average in $\mathrm{Eq}$. (62) and substituting in Eq. (57), together with Eq. (58), we obtain

$$
\begin{aligned}
\frac{\partial P(\mathbf{x}, t)}{\partial t}= & \frac{\partial}{\partial x_{j}}\left(\int_{0}^{t} d s \int d \mathbf{x}^{\prime} R_{j k}\left(\mathbf{x}, t ; \mathbf{x}^{\prime}, s\right)\right. \\
& \left.\times \frac{\partial}{\partial x_{k}}\left[\delta\left(\mathbf{x}-\mathbf{x}^{\prime}\right) P(\mathbf{x}, s)\right]\right) \\
& +\frac{\partial}{\partial x_{j}} \int d \mathbf{x}^{\prime} \int_{0}^{\infty} d s R_{j k}\left(\mathbf{x}, t ; \mathbf{x}^{\prime}, s\right) \frac{\partial}{\partial x_{m}} \\
& \times \int_{0}^{t} d u\left\langle f_{m}(x, u) \frac{\delta \rho[\mathbf{x}, u ; \mathbf{f}]}{\delta f_{k}\left(\mathbf{x}^{\prime}, s\right) d \mathbf{x}^{\prime} d s}\right\rangle .
\end{aligned}
$$

The iteration can be continued by applying Novikov's formula in the second term of Eq. (63); the resulting expression, as shown in Eq. (54), will involve derivatives of order higher than the second, and therefore we truncate the iteration in the lowest order. The following Fokker-Plancklike equation for $P(\mathrm{x}, t)$ is obtained

$$
\begin{aligned}
\frac{\partial P(\mathbf{x}, t)}{\partial t}= & \frac{\partial}{\partial x_{j}}\left(\int_{0}^{t} d s \int d \mathbf{x}^{\prime} R_{j k}\left(\mathbf{x}, t ; \mathbf{x}^{\prime}, s\right) \frac{\partial}{\partial x_{k}}\right. \\
& \left.\times\left[\delta\left(\mathbf{x}-\mathbf{x}^{\prime}\right) P(\mathbf{x}, s)\right]\right) .
\end{aligned}
$$

The time behavior of the first- and second-order moments of $P(x, t)$ are obtained by multiplying Eq. (64) by $X_{i}$ and $X_{i}^{2}$, respectively, and integrating over $\mathrm{x}$. We obtain

$$
\frac{d}{d t} \bar{X}_{i}(t)=\int_{0}^{t} d s \int d \mathbf{x}\left(\frac{\partial}{\partial x_{l}} R_{i l}\left(\mathbf{x}, t ; \mathbf{x}^{\prime}, s\right)\right)_{\mathbf{x}^{\prime}=\mathbf{x}} P(\mathbf{x}, s)
$$

and

$$
\begin{aligned}
\frac{d}{d t} \overline{X_{i}^{2}}(t)= & 2 \int_{0}^{t} d s \int d \mathbf{x}\left[R_{i i}(\mathbf{x}, t ; \mathbf{x}, s)\right. \\
& \left.+X_{i}\left(\frac{\partial}{\partial x_{l}} R_{i l}\left(\mathbf{x}, t ; \mathbf{x}^{\prime}, s\right)\right)_{\mathbf{x}^{\prime}=\mathbf{x}}\right] P(\mathbf{x}, s) .
\end{aligned}
$$

Here in Eq. (66) summation goes only over the index $l$.

The Eqs. (64)-(66) will be used to investigate the motion of the guiding center of charged particles in electric and magnetic fields, with random fluctuations both in space and time.

\section{c. On the accuracy of the iterative method}

Here we use the results previously obtained to illustrate the application of Eqs. (64)-(66) to study the diffusion of guiding centers when the fluctuations in the electric and magnetic fields are Gaussian random processes with zero mean, no space dependence, and all magnetic drifts are neglected. By solving this problem in an exact form we obtain a basic tool to test the accuracy of the lowest-order approximation of the iterative procedure shown in the last subsections. Within the above approximations, the guiding-center equations (22)-(25) reduce to,

$$
\frac{d X(t)}{d t}=V_{\|}(t) \frac{b_{x}(t)}{B_{0}}+c \frac{e_{y}(t)}{B_{0}}
$$

and

$$
\frac{d V_{\|}(t)}{d t}=-v_{c} V_{\|}(t)+\frac{q}{m} E_{0}+S(t),
$$

where

$$
S^{\prime}(t)=(q / m) e_{y}(t)+F(t) .
$$

We do not include the equation for $Y(t)$ since, because of symmetry, it should yield identical results to those corresponding to $X(t)$.

As shown in Appendix A, if we further assume that the random processes $\left\{b_{x}(t)\right\},\left\{e_{y}(t)\right\}$, and $\{s(t)\}$ are uncorrelated stationary Gaussian random processes with zero mean and autocorrelation functions given in Eq. (A3), the Eqs. (67) and (68) can be written as

$$
\begin{aligned}
& \frac{d}{d t} \delta x(t)=\left[\left\langle V_{\|}(t)\right\rangle+\delta V_{\|}(t)\right] \frac{b_{x}(t)}{B_{0}}+c \frac{e_{y}(t)}{B_{0}}, \\
& \frac{d}{d t} \delta V_{\|}(t)=-v_{c} \delta V_{\|}(t)+S(t),
\end{aligned}
$$

where $\quad \delta x(t)=x(t)-\langle x(t)\rangle \quad$ and $\quad \delta V_{\|}(t)=V_{\|}(t)$ $-\left\langle V_{\|}(t)\right\rangle$. The rhs of Eq. (70) has zero average as re- 
quired in the procedure we followed in the earlier sections, but the average of Eq. (71) does not vanish. By defining

$$
x_{1}(t)=\delta x(t)
$$

and

$$
x_{2}(t)=\delta V_{\|}(t) e^{v c t},
$$

we cast Eqs. (70) and (71) in the standard form, Eq. (34),

$$
\frac{d}{d t} x_{1}(t)=f_{1}\left(x_{1}, x_{2}, t\right)
$$

and

$$
\frac{d}{d t} x_{2}(t)=f_{2}\left(x_{1}, x_{2}, t\right),
$$

where

$$
f_{1}\left(x_{1}, x_{2}, t\right)=\left[\left\langle V_{\|}(t)\right\rangle+x_{2} e^{-v_{c} t}\right] \frac{b_{x}(t)}{B_{0}}+c \frac{e_{y}(t)}{B_{0}}
$$

and

$$
f_{2}\left(x_{1}, x_{2}, t\right)=S(t) e^{v_{c} t} .
$$

Here $\left\langle V_{\|}(t)\right\rangle$ is given in Eq. (A6). The averages of Eqs. (76) and (77) are zero as required; it also follows from these equations that

$$
\begin{aligned}
R_{x_{1} x_{1}}\left(\mathbf{x}, t ; \mathbf{x}^{\prime}, s\right)= & \left(1 / B_{0}^{2}\right)\left[\left\langle V_{\|}(t)\right\rangle\left\langle V_{\|}(s)\right\rangle+x_{2} x_{2}^{\prime} e^{-v_{c}(t+s)}\right] \\
& \times R_{b_{x}}(t-s)+\left(c^{2} / B_{0}^{2}\right) R_{e y}(t-s)
\end{aligned}
$$

$R_{x_{2} x_{2}}\left(\mathbf{x}, t ; \mathbf{x}^{\prime}, s\right)=e^{v_{c}(t+s)} R_{s}(t-s)$,

and

$$
R_{x_{1} x_{2}}\left(\mathbf{x}, t ; \mathbf{x}^{\prime}, s\right)=0,
$$

where again no correlations between $b_{x}$ and $e_{y}$ are taken into account.

Notice that if the electric and magnetic field random variations were not only time dependent but also space dependent, the space-time structure of the fluctuations should appear in the correlation functions $R_{b_{x}}, R_{e_{y}}$, and $R_{s}$ contained in Eqs. (78) and (79).

Using Eqs. (76) and (77) we obtain the first two moments

$$
\frac{d}{d t} \bar{x}_{1}(t)=\frac{d}{d t} \bar{x}_{2}(t)=0
$$

and

$$
\begin{aligned}
\frac{d}{d t} \overline{x_{1}^{2}}(t)= & \frac{2}{B_{0}^{2}} \int_{0}^{t} d s\left\{\left[\left\langle V_{\|}(t)\right\rangle\left\langle V_{\|}(s)\right\rangle+\overline{x_{2}^{2}}(s) e^{-v_{c}(t+s)}\right]\right. \\
& \left.\times R_{b_{x}}(t-s)+c^{2} R_{e_{y}}(t-s)\right\},
\end{aligned}
$$

together with

$$
\frac{d}{d t} \overline{x_{2}^{2}}(t)=2 \int_{0}^{t} d s e^{v_{c}(t+s)} R_{s}(t-s)
$$

Substituting Eq. (73) in Eq. (83) and integrating with respect to time, we reproduce the exact expression, Eq. (A13). The situation is different with Eq. (82), which differs from the exact expression, Eq. (A18). The difference lies in that $\overline{\delta V_{\|}(t) \delta V_{\|}}(s)$ in the exact form of $\overline{d x_{1}^{2}}(t) / d t$, is replaced by

$$
\overline{\delta V_{\|}(t) \delta V_{\|}}(s)=e^{-v_{c}(t-s)} \overline{\delta V_{\|}^{2}}(s) .
$$

This is equivalent to assuming $S(t)=0$ in Eq. (71) and writing $\delta V_{\|}(t)=e^{-v_{c}(t-s)} \delta V_{\|}(s)$ in the left-hand side (lhs) of Eq. (84).

The reason of this discrepancy is the following: In order to apply Novikov's theorem we assume that the rhs of Eq. (34) was a Gaussian stochastic vector process of zero mean. The rhs in Eq. (75), which is given in Eq. (77) is a Gaussian process with zero mean, which in turn yields $x_{2}(t)$ Gaussian; this however, since we are assuming $b_{x}(t)$ is Gaussian, implies that the second term in Eq. (76) is not Gaussian and therefore invalidates the use of the Novikov's formula. We should point out that the results would be correct if there were no stochastic term in the equation for $V_{\|}(t)$, setting $S(t) \equiv 0$ in Eq. (68). In the next section, we apply the results obtained in Sec. V B to study the effect of space and time stochastic fluctuations in the electric and magnetic field on the diffusion of guiding centers.

\section{DIFFUSION IN STOCHASTIC FIELDS WITH TIME AND SPACE DEPENDENCE}

In this section we study the problem of the diffusion of guiding centers induced by space and time fluctuations in the electric and magnetic fields using the theory developed in the last section. We shall, however, restrict ourselves to the case of a charged particle moving with a constant speed along the field lines, i.e., $V_{\|}=$const; this restriction is imposed here in order to validate Novikov's formula as was discussed in Sec. V C. Although we can take a deterministic time dependence in $V_{\|}$, we will simplify the equations even further by taking $V_{\|}$constant to show clearly the effect of stochastic space and time fluctuations of the fields on the diffusion of test particles. The equations of motion we consider are Eqs. (22) to (24), where the stochastic $\nabla B$ and curvature drifts are taken into account. To our knowledge, the analysis of the diffusion induced by these drifts has, at least in plasma fusion literature, never been done before. In addition we make two important simplifying assumptions: the first one is that the electric field is electrostatic, i.e.,

$$
\mathbf{e}(\mathbf{r}, t ; \widetilde{\omega})=-\nabla \Phi(\mathbf{r}, t ; \widetilde{\omega}),
$$

and the second is that the stochastic fluctuations constitute a Gaussian, stationary, homogeneous, and cylindrically symmetric turbulent field (see Appendix B). (This last property is known in the hydrodynamic literature as axisymmetric turbulence, ${ }^{32}$ however, we do not use this name here because in the tokamak jargon this has a different meaning and therefore it may lead to misunderstandings.) Within this type of turbulence the autocorrelation and 
cross-correlation functions of $\mathbf{b}$ and $\Phi$ take some simple analytical forms, as shown in Appendix B. The conditions of homogeneity and cylindrical symmetry may hold in tokamaks with large-aspect ratio and the equations of motion we are using are valid for passing particles only (some analysis of the diffusion of trapped particles induced by stochastic electromagnetic fields is found in Ref. 13). Further, although no correlation between electrostatic and magnetic fluctuations were seen in early experiments, ${ }^{1}$ more recent reports ${ }^{3}$ show the appearance of this correlation at high frequencics. Therefore, in what follows we keep this correlation as different from zero.

For describing the motion of guiding centers in stochastic electric and magnetic fields we take the Eqs. (22) and (23) with $V_{\|}$and $V_{1}$ constant. The probability density function depends now only on $x, y$, and $t$, i.e., $P(x, y, t)$ since $z$ is a function only of time, i.e.,

$$
z=V_{\|} t
$$

Equations (22) and (23) are written in the standard form of Eq. (34) as

$$
\begin{aligned}
& \frac{d x(t)}{d t}=f_{x}[x, y, z(t) ; \tilde{\omega}], \\
& \frac{d y(t)}{d t}=f_{y}[x, y, z(t) ; \tilde{\omega}],
\end{aligned}
$$

where $f_{x}$ and $f_{y}$ are given by the rhs of Eqs. (22) and (23). We can now use Eqs. (65) and (66) to calculate the time behavior of the first- and second-order moments of $x$ and $y$. From them, the diffusion coefficient can be obtained as $D_{\perp}=\frac{1}{2} d x^{2}(t) / d t$. To do this, however, we need first to evaluate the correlations

$$
R_{u v}(\xi, \tau)=\left\langle f_{u}(\mathbf{x}, i ; \widetilde{\omega}) f_{v}\left(\mathbf{x}^{\prime}, t^{\prime} ; \tilde{\omega}\right)\right\rangle,
$$

where $\xi=\mathrm{x}-\mathrm{x}^{\prime}, \tau=t-t^{\prime}$, and $u, v=x$ or $y$. The fundamental correlation functions appearing in the functions $\left\{R_{i j}\right\}$ are:

$$
\begin{aligned}
& \beta_{i j}(\xi, \tau)=\left\langle b_{i}(\mathbf{x}, t) b_{j}\left(\mathbf{x}^{\prime}, t^{\prime}\right)\right\rangle, \\
& L_{i}^{(1)}(\xi, \tau)=\left\langle\Phi(\mathbf{x}, t) b_{i}\left(\mathbf{x}^{\prime}, t^{\prime}\right)\right\rangle, \\
& L_{i}^{(2)}(\xi, \tau)=\left\langle b_{i}(\mathbf{x}, t) \Phi\left(\mathbf{x}^{\prime}, t^{\prime}\right)\right\rangle,
\end{aligned}
$$

and

$$
\psi(\xi, \tau)=\left\langle\Phi(\mathbf{x}, t) \Phi\left(\mathbf{x}^{\prime}, t^{\prime}\right)\right\rangle,
$$

with $i, j=x, y$, or $z$. It is worthwhile to point out that although the probability density function $P(x, y, t)$ does not involve $z$ (because of the assumption $V_{\|}=$const) the guiding-center equations involve a $z$ dependence (in the $\nabla B$ and curvature drift terms) that should be taken into account in the corresponding correlations before substituting $z=V_{\|} t$. In general there are nine functions $\beta_{i j}$, three functions $L_{i}^{(1)}$ or $L_{l}^{(2)}$ and one function $\psi$. In the case of cylindrically symmetric turbulence with the axis of symmetry pointing in the $\hat{z}$ direction, which is the direction of our average magnetic field $\mathbf{B}_{0}$, it follows that [see Appendix $\mathbf{B}$, Eq. (B15)] $\beta_{i j}$

$$
=\left(\begin{array}{ccc}
\beta_{0} \xi_{x}^{2}+\beta_{1} & \beta_{0} \xi_{x} \xi_{y} & \beta_{0} \xi_{x} \xi_{z}+\beta_{3} \xi_{x} \\
\beta_{0} \xi_{y} \xi_{x} & \beta_{0} \xi_{y}^{2}+\beta_{1} & \beta_{0} \xi_{y} \xi_{z}+\beta_{3} \xi_{y} \\
\beta_{0} \xi_{z} \xi_{x}+\beta_{3} \xi_{x} & \beta_{0} \xi_{z} \xi_{y}+\beta_{3} \xi_{y} & \beta_{0} \xi_{z}^{2}+\beta_{1}+\beta_{2}+2 \beta_{3} \xi_{z}
\end{array}\right) .
$$

Here $\beta_{0}, \beta_{1}$, and $\beta_{2}$ are even functions in $\xi^{2}, \tau$, and $\xi_{z}$, and $\beta_{3}$ is an even function in $\xi^{2}$ and $\tau$, but an odd function in $\xi_{z}$. Thus, in this case $\beta_{i j}$ contains only four different functions instead of nine functions. Moreover, from the condition $\boldsymbol{\nabla} \cdot \mathbf{b}=0$ it follows that [see Appendix B, Eqs. (B11)]

$$
\frac{\partial \beta_{i j}}{\partial \xi_{i}}=0 \text { and } \frac{\partial \beta_{i j}}{\partial \xi_{j}}=0,
$$

reducing therefore the number of independent functions to two. From Eq. (B21), we have

$$
L_{i}^{(\alpha)}=M^{(\alpha)} \xi_{i}+N^{(\alpha)} \delta_{i z},
$$

with $\alpha=1$ or 2 . It holds that $M^{(1)}\left(\xi^{2}, \xi_{z}, \tau\right)$ $=-M^{(2)}\left(\xi^{2},-\xi_{z}, \tau\right)$ and $N^{(1)}\left(\xi^{2}, \xi_{z}, \tau\right)=N^{(2)}\left(\xi^{2},-\xi_{z}, \tau\right)$ The condition $\boldsymbol{\nabla} \cdot \mathbf{b}=0$ implies that [see Eq. (B25)]

$$
\frac{\partial L_{i}^{(\alpha)}}{\partial \xi_{i}}=0
$$

It is shown in Appendix B that $\psi\left(\xi^{2}, \xi_{z} \tau\right)$, defined in Eq. (91), is an even function in all its arguments.

In addition to all the above requirements for the correlation functions of cylindrically symmetric turbulent fields, there are other general conditions to be satisfied by any function regardless the symmetry that should also be taken into account, ${ }^{30}$ e.g., the autocorrelation functions should have a maximum at $\xi=0$ and $\tau=0$.

By using Eqs. (88), (87), and (22) $R_{x x}$ turns out to be

$$
\begin{aligned}
R_{x x}= & \frac{V_{\|}^{2}}{B_{0}^{2}} \beta_{x x}+\frac{V_{\|} c}{B_{0}^{2}} \frac{\partial\left[L_{x}^{(1)}-L_{x}^{(2)}\right]}{\partial \xi_{y}}-\frac{c^{2}}{B_{0}^{2}} \frac{\partial^{2} \psi}{\partial \xi_{y}^{2}} \\
& -\frac{c V_{1}^{2}}{B_{0}^{2} \omega_{g}} \frac{\partial^{2}\left[L_{z}^{(1)}+L_{z}^{(2)}\right]}{\partial \xi_{y}^{2}}-\frac{c V_{\|}^{2}}{B_{0}^{2} \omega_{g}} \frac{\partial^{2}\left[L_{y}^{(1)}+L_{y}^{(2)}\right]}{\partial \xi_{y} \partial \xi_{z}} \\
& -\frac{V_{\perp}^{4}}{\omega_{g}^{2} B_{0}^{2}} \frac{\partial^{2} \beta_{z z}}{\partial \xi_{y}^{2}}-\frac{V_{1}^{2} V_{\|}^{2}}{B_{0}^{2} \omega_{g}^{2}} \frac{\partial^{2}\left[\beta_{z y}+\beta_{y z}\right]}{\partial \xi_{y} \partial \xi_{z}}-\frac{V_{\|}^{4}}{B_{0}^{2} \omega_{g}^{2}} \frac{\partial^{2} \beta_{y y}}{\partial \xi_{z}^{2}},
\end{aligned}
$$

together with the corresponding expressions for $R_{y y}, R_{x y}$, and $R_{y x}$.

With those expressions we can then calculate $\bar{x}(t)$ and $\bar{y}(t)$ from Eq. (65). In doing this we should first evaluate $\left(\partial R_{x x} / \partial \xi_{x}+\partial R_{x y} / \partial \xi_{y}\right)$ and $\left(\partial R_{y x} / \partial \xi_{x}+\partial R_{y y} / \partial \xi_{y}\right)$ at $\xi_{z}=V_{\|} \tau$ and $\xi_{x}=\xi_{y}=0$, and then integrate over $x, y$, and $\tau$. After a little lengthy but straightforward calculations we find that 


$$
\left.\frac{\partial R_{x x}}{\partial \xi_{u}}\right|_{\xi=\xi_{0}}=\left.\frac{\partial R_{x y}}{\partial \xi_{u}}\right|_{\xi=\xi_{0}}=\left.\frac{\partial R_{y x}}{\partial \xi_{u}}\right|_{\xi=\xi_{0}}=\left.\frac{\partial R_{y y}}{\partial \xi_{u}}\right|_{\xi=\xi_{0}}=0,
$$

where $\xi_{0} \equiv\left(0,0, V_{\|} \tau\right)$ and $u=x$ or $y$. It follows that

$$
\frac{d \bar{x}}{d t}=\frac{d \bar{y}}{d t}=0,
$$

as was expected due to the symmetry and the vanishing fluctuation average. On the other side, due to the same symmetry, the expressions for $\overline{y^{2}(t)}$ and $\overline{x^{2}(t)}$ should be identical, and hence we concentrate only on this second quantity. After application of Eq. (66) we obtain

$$
\frac{\overline{d x^{2}}}{d t}=2 \int_{0}^{t} d \tau R_{x x}\left(\xi=\xi_{0}, \tau\right)
$$

from which it follows that

$$
\begin{aligned}
\frac{d \overline{x^{2}}}{d t}= & 2 \int_{0}^{t} d \tau\left\{\frac{V_{\|}^{2}}{B_{0}^{2}} \beta_{1}\left(\xi=\xi_{0}, \tau\right)-\frac{2 c^{2}}{B_{0}^{2}}\left(\frac{\partial \psi}{\partial \omega}\right)_{\xi=\xi_{0}}-\frac{2 V_{\perp}^{4}}{B_{0}^{2} \omega_{g}^{2}}\left[\frac{\partial\left(\beta_{1}+\beta_{2}\right)}{\partial \omega}+V_{\|}^{2} \tau^{2} \frac{\partial \beta_{0}}{\partial \omega}+2 V_{\|} \tau\left(\frac{\partial \beta_{3}}{\partial \omega}\right)\right]_{\xi=\xi_{0}}-\frac{V_{\|}^{4}}{B_{0}^{2} \omega_{g}^{2}}\left(\frac{\partial^{2} \beta_{1}}{\partial \xi_{z}^{2}}\right)_{\xi=\xi_{0}}\right. \\
& -\frac{2 V_{1}^{2} V_{\|}^{2}}{B_{0}^{2} \omega_{g}^{2}}\left[\beta_{0}\left(\xi=\xi_{0}, \tau\right)+V_{\|} \tau \frac{\partial \beta_{0}}{\partial \xi_{z}}+\frac{\partial \beta_{3}}{\partial \xi_{z}}\right]_{\xi=\xi_{0}}-\frac{2 c V_{\perp}^{2}}{B_{0}^{2} \omega_{g}}\left[V_{\|} \tau \frac{\partial\left(M^{(1)}+M^{(2)}\right)}{\partial \omega}+\frac{\partial\left(N^{(1)}+N^{(2)}\right)}{\partial \omega}\right]_{\xi=\xi_{0}} \\
& \left.-\frac{c V_{\|}^{2}}{B_{0}^{2} \omega_{g}}\left[\frac{\partial\left(M^{(1)}+M^{(2)}\right)}{\partial \xi_{z}}\right]_{\xi=\xi_{0}}\right\}
\end{aligned}
$$

where

$$
\omega \equiv \xi_{x}^{2}+\xi_{y}^{2}
$$

By identifying the origin of the different terms appearing in the expression for the diffusion coefficient $D_{\perp}$ $=\frac{1}{2} d \bar{x}^{2} / d t$, we have that the diffusion resulting from the motion of particles along the fluctuating magnetic field, i.e., $V_{\|}\left(\mathbf{b} / B_{0}\right)$, gives

$$
D_{\perp}^{(1)}(t)=\frac{V_{\|}^{2}}{B_{0}^{2}} \int_{0}^{t} d \tau \beta_{1}\left(\xi=\xi_{0}, \tau\right) .
$$

This term is the equivalent to the first term in the rhs of Eq. (28) when $V_{\|}=$const and the space fluctuations are included. The diffusion coming from the autocorrelation of the electric drift $\mathbf{e} \times \mathbf{B}_{0}$ yields

$$
D_{1}^{(2)}(t)=-\frac{2 c^{2}}{B_{0}^{2}} \int_{0}^{t} d \tau\left(\frac{\partial \psi}{\partial \omega}\right)_{\xi=\xi_{0}},
$$

and is equivalent to the last term on the rhs of Eq. (28), but now the spatial variations of the fields were taken into account.

The diffusion induced from the autocorrelation of the $\nabla B$ drift, $\mathbf{B}_{0} \times \nabla b_{z}$, is

$$
\begin{aligned}
D_{1}^{(3)}(t)= & -\frac{2 V_{1}^{4}}{\omega_{g}^{2} B_{0}^{2}} \int_{0}^{t} d \tau\left(\frac{\partial\left(\beta_{1}+\beta_{2}\right)}{\partial \omega}+V_{\|}^{2} \tau^{2} \frac{\partial \beta_{0}}{\partial \omega}\right. \\
& \left.+2 V_{\|} \tau \frac{\partial \beta_{3}}{\partial \omega}\right)_{\xi=\xi_{0}} .
\end{aligned}
$$

The contributing part due to the autocorrelation of the curvature drift, i.e., $\mathbf{B}_{0} \times \partial \mathbf{b} / \partial z$, is

$$
D_{\perp}^{(4)}(t)=-\frac{V_{\|}^{4}}{B_{0}^{2} \omega_{g}^{2}} \int_{0}^{t} d \tau\left(\frac{\partial^{2} \beta_{1}}{\partial \xi_{z}^{2}}\right)_{\xi=\xi_{0}} .
$$

There are several terms coming from the cross correlations. One of them is that from the correlations between $\nabla B$ and curvature drifts, and is given by

$$
D_{\perp}^{(5)}(t)=-\frac{2 V_{\perp}^{2} V_{\|}^{2}}{B_{0}^{2} \omega_{g}^{2}} \int_{0}^{t} d \tau\left(\beta_{0}+V_{\|} \tau \frac{\partial \beta_{0}}{\partial \xi_{z}}+\frac{\partial \beta_{3}}{\partial \xi_{z}}\right)_{\xi=\xi_{0}} .
$$

From the correlation between electric and $\nabla B$ drifts it follows that

$$
\begin{aligned}
D_{\perp}^{(6)}(t)= & -\frac{2 c V_{\perp}^{2}}{B_{0}^{2} \omega_{g}} \int_{0}^{t} d \tau\left(V_{\|} \tau \frac{\partial\left(M^{(1)}+M^{(2)}\right)}{\partial \omega}\right. \\
& \left.+\frac{\partial\left(N^{(1)}+N^{(2)}\right)}{\partial \omega}\right)_{\xi=\xi_{0}},
\end{aligned}
$$

while from electric and curvature drifts it follows that

$$
D_{\perp}^{(7)}(t)=-\frac{c V_{\|}^{2}}{B_{0}^{2} \omega_{g}} \int_{0}^{t} d \tau\left(\frac{\partial\left(M^{(1)}+M^{(2)}\right)}{\partial \xi_{z}}\right)_{\xi=\xi_{0}} .
$$

We can notice that as a consequence of the quasilinear approximation (i.e., $\xi_{x}=\xi_{y}=0$ ) and the analytical form of the cross correlation $\left\langle b_{x} e_{y}\right\rangle$ in cylindrically symmetric tur- 
bulence [see Eqs. (90) and (94)], the expected diffusion due to the term $\left\langle b_{x} e_{y}\right\rangle$ vanishes in Eq. (100). In the same way the diffusion expected from the correlations of $\mathbf{b}$ with $\nabla B$ and the curvature drift vanishes.

\section{SUMMARY AND CONCLUSIONS}

In this paper we have studied the diffusion of charged particles in a uniform strong magnetic field with prescribed stochastic magnetic and electric fluctuations with both space and time dependence. We have analyzed the guidingcenter equations to be used for fluctuations observed in tokamaks, and found that the stochastic curvature and $\nabla B$ drifts resulting from the space dependence of the magnetic fluctuations may become important for fluctuations with large wave numbers. As a benchmark for our treatment we have revised the case of fluctuations without space dependence, neglecting therefore the magnetic drifts, but using an exact treatment to calculate the diffusion coefficients. In this case, the diffusion due to the fluctuations in the magnetic field lines is equivalent to the diffusion in frozen turbulence, because the time and the space coordinate along the direction of the homogeneous magnetic field play essentially the same role.

In order to evaluate the diffusion coefficient in the general case of fluctuations with time and space dependence we have used Novikov's theorem, which requires a Gaussian distribution for the stochastic fields. This consequently limits our model to the case $V_{\|}=$const. Up to this point the results were independent of any specific structure of the turbulent fields; however, to go further in our calculations, we have introduced the assumption of homogeneous cylindrically symmetric turbulence and obtained the diffusion coefficient for this specific turbulent structure, which now comprises both time and space dependence. The several terms composing this coefficient can be identified by their origins. The term $D_{1}^{(1)}$ in Eq. (101a) describes the diffusion of guiding centers due to their motion along stochastic field lines. This diffusion coefficient can be evaluated for two limiting cases: one is the case without space dependence in which $D_{1}^{(1)}$ reproduces the first term on the right-hand side of Eq. (28), after $V_{\|}=$const is assumed, and the other case is the frozen turbulence, where the diffusion of magnetic lines is given by $D_{m}=\left\langle b_{x}(0) b_{x}(0)\right\rangle \lambda_{\|} / B_{0}^{2}$. Here $D_{m}$ can be obtained from $D_{1}^{(1)}$ after canceling the explicit time dependence and replacement of the effective correlation time $\tau_{\perp b}$ [defined as in Eq. (29a)] by $\tau_{\perp b}=\lambda_{\|} / V_{\|}$. The remaining difference between $D_{m}$ and $D_{1}^{(1)}$ is the factor $V_{1}^{2}$, which accounts for the difference between magnetic line diffusion and particle diffusion. We notice in Eq. (101a) that for diffusion process purposes a particle cannot differentiate between remaining at a fixed position (subject to fluctutations in time) and traveling along frozen fluctuations. This can be seen by considering the change of variable $\tau$ by $V_{\|} \tau$ in the two cases mentioned above. In general, however, a combination of both effects is present.

The diffusion of guiding centers due to the stochastic electric drift $\mathbf{e} \times \mathbf{B}_{0}$ is given by Eq. (101b) in terms of the autocorrelation of the electric potential fluctuations. In general, this term $D_{\mathrm{I}}^{(2)}$ is believed to be the most important in the diffusion induced by stochastic electromagnetic fluctuations in tokamaks, since the magnetic fluctuations are very small [see Eqs. (32) and (33)]. ${ }^{2}$ This diffusion coefficient does not depend either on the electric charge or on the mass of the particles and, in the case the fluctuation properties do not depend on $B_{0}$, the coefficient shows the characteristic $B^{-2}$ dependence.

The expressions $D_{1}^{(3)}$ and $D_{I}^{(4)}$ in Eqs. (101) give the diffusion coefficient associated to the autocorrelation of the $\nabla B$ curvature and stochastic drifts, respectively. These terms become important for magnetic fluctuations with large wave numbers. They have a strong dependence on the particle mass and on the parallel and the perpendicular components of the particle velocity that can be written as $\left(m V_{1}^{2}\right)^{2}$ and $\left(m V_{\|}^{2}\right)^{2}$, which roughly gives the square of the perpendicular and the parallel temperature for the case of a Gaussian distribution. The dependence of $D_{1}^{(4)}$ on $V_{\|}$is seen to be very important when a loop voltage is present and the plasma has a low resistivity.

In order to evaluate the relative importance of the diffusion induced by stochastic magnetic drifts further work is needed in modeling the correlation functions $\beta_{0}, \beta_{1}, \beta_{2}$, and $\beta_{3}$; this will be the subject of a future paper.

We should make it clear that from our formulas for the diffusion coefficients, no scaling laws can be deduced unless we give the scaling of the statistical properties of the fluctuations. This would require a model for the turbulence or a wide range of experimental measurements in different tokamaks and under different operating conditions. We should also mention that, within the frame of our formulation, only diffusive flows (due to density inhomogeneities) can be considered, and the problem of an inward particle convective flow of the type appearing in the empirical transport formulas for tokamaks cannot be addressed.

Finally we should briefly discuss the ambipolarity issue. From the expression (101) we see that except for the electric drift diffusion $D_{1}^{(2)}$ all the terms in $D_{\perp}$ give, in general, different results for electrons and ions, which shows that diffusion is not intrinsically ambipolar. This indicates that our assumptions on the fluctuations are not intrinsically consistent with charge conservation, i.e., $\boldsymbol{\nabla \cdot J}$ $=0$, and thus ambipolarity should be imposed externally. It is, however, questionable to require ambipolarity at this level, since the diffusion mechanisms we are considering in this paper are only a component of the total transport processes occurring in the plasma, which logically should satisfy ambipolarity in a global sense. Hence, the main purpose of our calculations is to provide a picture of the magnitude and importance of the diffusion mechanisms we are considering and, in that respect, contribute to the search for the main mechanisms responsible for the transport in tokamaks. After a clear idea of these mechanisms prevails, a self-consistent calculation of the plasma turbulence would provide a meaningful intrinsic ambipolar clectric field. 


\section{APPENDIX A: FLUCTUATIONS WITHOUT SPACE DEPENDENCE}

In this appendix we shall find the exact expressions for the second-order moments of $x$ and $V_{\|}$when they obey the following stochastic differential equations:

$$
\frac{d x(t)}{d t}=V_{\|} \frac{b_{x}(t)}{B_{0}}+c \frac{e_{y}(t)}{B_{0}}
$$

and

$$
\frac{d V_{\|}(t)}{d t}=-v_{c} V_{\|}+\frac{q}{m} E_{0}+s(t),
$$

where $s(t)=(q / m) e_{z}(t)+F(t)$; here $b_{x}, e_{y}$, and $s(t)$ are stationary uncorrelated random fluctuations with zero means and

$$
\begin{aligned}
& \left\langle b_{x}(t) b_{x}(t+\tau)\right\rangle=R_{b_{x}}(|\tau|), \\
& \left\langle e_{y}(t) e_{y}(t+\tau)\right\rangle=R_{e_{y}}(|\tau|),
\end{aligned}
$$

and

$$
\langle s(t) s(t+\tau)\rangle=R_{s}(|\tau|) .
$$

With the above assumptions we have that the processes $V_{\|}(t)$ and $b_{x}(t)$ are also uncorrelated. By taking averages in Eqs. (A1) and (A2) we have

$$
\begin{aligned}
& \frac{d}{d t}\langle x(t)\rangle=0, \\
& \frac{d}{d t}\left\langle V_{\|}(t)\right\rangle=-v_{c}\left\langle V_{\|}\right\rangle+\frac{q}{m} E_{0},
\end{aligned}
$$

from which it follows that $\langle x(t)\rangle$ is constant and

$$
\left\langle V_{\|}(t)\right\rangle=V_{0} e^{v_{c} t}+\left(q E_{0} / m v_{c}\right)\left(1-e^{v_{c} t}\right) .
$$

Here we have assumed that at time $t=0$ all the particles have the same velocity $V_{0}$. Substracting Eqs. (A4) and (A5) from (A1) and (A2), respectively, and defining $\delta x(t)=x(t)-\langle x(t)\rangle$ and $\delta V_{\|}(t)=V_{\|}(t)-\left\langle V_{\|}(t)\right\rangle$ we obtain

$$
\frac{d}{d t} \delta x(t)=\left[\left\langle V_{\|}(t)\right\rangle+\delta V_{\|}(t)\right] \frac{b_{x}(t)}{B_{0}}+c \frac{e_{y}(t)}{B_{0}},
$$

and

$$
\frac{d}{d t} \delta V_{\|}(t)=-v_{c} \delta V_{\|}(t)+s(t) .
$$

Integrating (A8) we have

$$
\delta V_{\|}(t)=e^{-v_{c} l} \int_{0}^{t} d u e^{v_{c} u} s(u)
$$

the autocorrelation function $R_{V_{\|}}\left(t, t^{\prime}\right)$ $=\left\langle\delta V_{\|}(t) \delta V_{\|}\left(t^{\prime}\right)\right\rangle$ is given by

$R_{V_{\|}}\left(t, t^{\prime}\right)=e^{-v_{c}\left(t+t^{\prime}\right)} \int_{0}^{t} d u \int_{0}^{t^{\prime}} d u^{\prime} e^{v_{c}\left(u+u^{\prime}\right)} R_{s}\left(\left|u-u^{\prime}\right|\right) ;$

thus, we have when $t \geqslant t^{\prime}$

$$
\begin{aligned}
R_{V_{\|}}\left(t, t^{\prime}\right)= & \frac{1}{2 v_{c}}\left(e^{-v_{c}\left(t-t^{\prime}\right)} \int_{-\left(t-t^{\prime}\right)}^{t^{\prime}} d u R_{s}(|u|) e^{-v_{c} u}\right. \\
& \left.+e^{v_{c}\left(t-t^{\prime}\right)} \int_{\left(t-t^{\prime}\right)}^{t} d u R_{s}(|u|) e^{-v_{c} u}\right) \\
& -\frac{1}{2 v_{c}} e^{-v_{c}\left(t+t^{\prime}\right)}\left(\int_{0}^{t^{\prime}} d u R_{s}(|u|) e^{v_{c} u}\right. \\
& \left.+\int_{0}^{t} d u R_{s}(|u|) e^{v_{c} u}\right),
\end{aligned}
$$

and when $t, t^{\prime} \rightarrow \infty$ with $t-t^{\prime}=\tau$ we get from Eq. (A11) that the process $\delta V_{\|}$becomes stationary with autocorrelation function given by

$$
R_{V_{\|}}(\tau)=\frac{1}{2 v_{c}} \int_{-\infty}^{\infty} d u e^{-v_{c}|u-\tau|} R_{s}(|u|) .
$$

It is interesting to evaluate $R_{V_{\|}}(t, t)=\left\langle\delta V_{\|}^{2}(t)\right\rangle$ since this quantity describes the so-called stochastic heating, which is an increment of the parallel energy of the guiding centers due to a parallel stochastic electric field [see Eq. (A2)]. It also gives the diffusion coefficient in velocity space.

The total parallel energy of the particle is proportional to

$$
\left\langle V_{\|}^{2}(t)\right\rangle=\left\langle V_{\|}(t)\right\rangle^{2}+\left\langle\delta V_{\|}^{2}(t)\right\rangle,
$$

with $\left\langle V_{\|}(t)\right\rangle$ given by Eq. (A6) and $\left\langle\delta V_{\|}^{2}(t)\right\rangle$ from Eq. (A11), we obtain

$$
\left\langle\delta V_{\|}^{2}(t)\right\rangle=\frac{1}{v_{c}} e^{-v_{c} t} \int_{0}^{t} d u R_{s}(|u|)\left(e^{v_{c}(t-u)}-e^{-v_{c}(t-u)}\right) .
$$

For $t \ll v_{c}^{-1}$ this quantity increases approximately linearly in time as

$$
\left\langle\delta V_{\|}^{2}(t)\right\rangle=2 t \int_{0}^{t} d u\left(1-\frac{u}{t}\right) R_{s}(|u|),
$$

and, due to collisions, for $t>v_{c}^{-1}$ it reaches a constant value given by

$$
\left\langle\delta V_{\|}^{2}(t)\right\rangle=\frac{1}{v_{c}} \int_{0}^{\infty} d u R_{s}(|u|) e^{-v_{c} u} .
$$

The diffusion coefficient in velocity space is defined as $D_{V_{\|}}(t)=\frac{1}{2} d\left\langle\delta V_{\|}^{2}(t)\right\rangle / d t$ so that

$$
D_{V_{\|}}(t)=e^{-2 v_{c} t} \int_{0}^{t} d u R_{s}(|u|) e^{v_{c} u}
$$

For $t \ll v_{c}^{-1}$ it increases in time, later $D_{V_{\|}}$reaches a maximum and for much larger times, as a consequence of collisions, it reduces to zero. Without collisions the diffusion coefficient increases continuously and reaches a constant value for $t \gg \tau_{\text {corr }}$.

We can now return to Eq. (A7) and integrate it with respect to time

$$
\delta x(t)=\int_{0}^{t} d t^{\prime}\left(\left[\left\langle V_{\|}\left(t^{\prime}\right)\right\rangle+\delta V_{\|}\left(t^{\prime}\right)\right] \frac{b_{x}\left(t^{\prime}\right)}{B_{0}}+c \frac{e_{y}\left(t^{\prime}\right)}{B_{0}}\right),
$$


the autocorrelation function $R_{x x}\left(t, t^{\prime}\right)=\left\langle\delta x(t) \delta x\left(t^{\prime}\right)\right\rangle$ is obtained from Eq. (A16) as

$$
\begin{aligned}
R_{x}\left(t, t^{\prime}\right)= & \frac{1}{B_{0}^{2}} \int_{0}^{t} d \tau_{1} \int_{0}^{t^{\prime}} d \tau_{2}\left[\langle V _ { \| } ( \tau _ { 1 } ) \rangle \langle V _ { \| } ( \tau _ { 2 } ) \rangle R _ { b _ { x } } \left(\mid \tau_{1}\right.\right. \\
& \left.-\tau_{2} \mid\right)+\left\langle\delta V_{\|}\left(\tau_{1}\right) \delta V_{\|}\left(\tau_{2}\right)\right\rangle R_{b_{x}}\left(\left|\tau_{1}-\tau_{2}\right|\right) \\
& \left.+c^{2} R_{e_{y}}\left(\left|\tau_{1}-\tau_{2}\right|\right)\right],
\end{aligned}
$$

where $\left\langle\delta V_{\|}\left(\tau_{1}\right) \delta V_{\|}\left(\tau_{2}\right)\right\rangle$ is given by Eq. (A11). In order to get the diffusion coefficient in configuration space we evaluate $\mathrm{Eq}$. (A17) at $t^{\prime}=t$ and, after differentiating with respect to time, we obtain

$$
\begin{aligned}
D_{1}(t)= & \frac{1}{2} \frac{d}{d t}\left\langle\delta x^{2}(t)\right\rangle \\
= & \frac{1}{B_{0}^{2}}\left\langle V_{\|}(t)\right\rangle \int_{0}^{t} d \tau\left\langle V_{\|}(t-\tau)\right\rangle R_{b_{x}}(|\tau|) \\
& +\frac{1}{B_{0}^{2}} \int_{0}^{t} d \tau\left\langle\delta V_{\|}(t) \delta V_{\|}(t-\tau)\right\rangle R_{b_{x}}(|\tau|) \\
& +\frac{c^{2}}{B_{0}^{2}} \int_{0}^{t} d \tau R_{e_{y}}(|\tau|) .
\end{aligned}
$$

The asymptotic value of the diffusion coefficient is obtained from Eq. (A18) by taking the limit $t \rightarrow \infty$. If the magnetic field fluctuations $b_{x}(t)$ have finite correlation time, we can approximate the asymptotic value of the diffusion coefficient as

$$
\begin{aligned}
D_{1}(t \rightarrow \infty) & =\left(\frac{q E_{0}}{m v_{c} B_{0}}\right)^{2} \int_{0}^{\infty} d \tau R_{b_{x}}(|\tau|)+\frac{1}{2 v_{c} B_{0}^{2}} \int_{0}^{\infty} d \tau \\
& \times \int_{-\infty}^{\infty} d u e^{-v_{c}|u-\tau|} R_{s}(|u|) R_{b_{x}}(|\tau|) \\
& +\frac{c^{2}}{B_{0}^{2}} \int_{0}^{\infty} d \tau R_{e_{y}}(|\tau|) .
\end{aligned}
$$

We will use expressions (A18) and (A19) in the main part of our paper to analyze the accuracy of the Novikov's method.

\section{APPENDIX B: STATIONARY HOMOGENEOUS TURBULENCE WITH CYLINDRICAL SYMMETRY}

In this appendix we present results concerning correlation functions of a stationary homogeneous turbulent field $\mathbf{u}$ in the presence of a preferred direction that establishes a cylindrical symmetry. This is the case considered in Sec. VI of the paper, i.e., a stochastic magnetic (electric) field superposed on a strong uniform magnetic (electric) field. In this derivation we follow the original works of Bachelor. $^{32,33}$

Let us consider the correlation function

$$
R_{i j}\left(\mathbf{x}, t ; \mathbf{x}^{\prime} t^{\prime}\right)=\left\langle u_{i}(\mathbf{x}, t ; \widetilde{\omega}) v_{j}\left(\mathbf{x}^{\prime}, t^{\prime} ; \widetilde{\omega}\right)\right\rangle,
$$

where $u_{i}(\mathrm{x}, t ; \widetilde{\omega})$ and $v_{j}\left(\mathrm{x}^{\prime}, t^{\prime}, \widetilde{\omega}\right)$ are the $i$ th and $j$ th component of the vector fields $u$ and $v$, respectively, and $\widetilde{\omega}$ is the

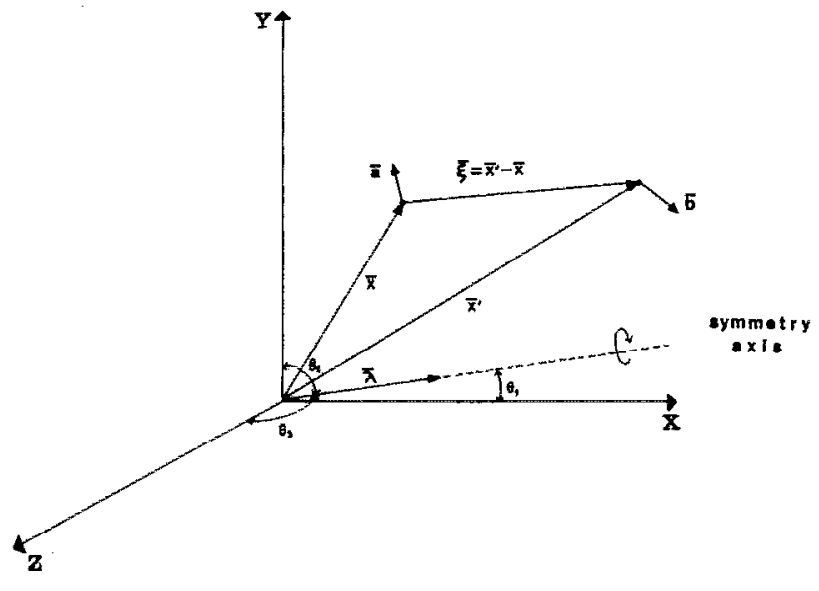

FIG. 1. Cylindrically symmetric turbulence. The diagram shows the axis of symmetry $\lambda$, and the vectors $\mathbf{a}$ and $\mathbf{b}$ at position $\mathbf{x}$ and $\mathbf{x}^{\prime}$, respectively.

label characterizing a certain realization of the stochastic process. Here $\langle\cdots\rangle$ means ensemble average.

Let us consider the following scalar quantity defined as the ensemble average of the product of $\mathbf{a} \cdot \mathbf{u}$ and $\mathbf{b} \cdot \mathbf{v}$,

$$
R_{0}(\mathbf{a}, \mathbf{b})=\left\langle a_{i} u_{i} b_{j} v_{j}\right\rangle=R_{i j} a_{i} b_{j},
$$

where $\mathbf{a}$ and $\mathbf{b}$ are two arbitrary constant vectors and $R_{i j}$ is the correlation function defined in Eq. (B1).

If we define the symmetry axes by the unit vector $\lambda$, we notice that, due to homogeneity, $R_{0}$ does not depend on $\mathrm{x}$ and $\mathrm{x}^{\prime}$ separately, but on $\xi=x^{\prime}-\mathbf{x}$. Also from the stationarity condition, it follows that $R_{0}$ should depend on the time difference $\tau=t^{\prime}-t$ and not on $t$ and $t^{\prime}$ explicitly. In addition, cylindrical symmetry implies that the function $R_{0}$ should remain unchanged by an arbitrary rotation around the axes of symmetry and also by reflections through planes that are perpendicular to $\lambda$ (see Fig. 1). This means that $R_{0}(\xi, \lambda, \mathbf{a}, \mathbf{b})$ must be a function only of fundamental invariants satisfying the same rotation and reflection conditions. These invariants are the set of scalar products formed by any two vectors $\xi, \lambda, \mathbf{a}$, and $\mathbf{b}$. Thus the most general form of $R_{0}$ is obtained by choosing the appropriate combinations of these scalar products to build $R_{i j} a_{i} b_{j}$. The arguments presented above concerning the construction of the scalar $R_{0}$ in Eq. (B2) can be applied for the construction of ensemble averages of the type

$$
R_{i j k}\left(\mathbf{x}, t ; \mathbf{x}^{\prime}, t^{\prime} ; \mathbf{x}^{\prime \prime}, t^{\prime \prime}\right)=\left\langle u_{i}(\mathbf{x}, t) v_{j}\left(\mathbf{x}^{\prime}, t^{\prime}\right) w_{k}\left(\mathbf{x}^{\prime \prime}, t^{\prime \prime}\right)\right\rangle
$$

and higher-order correlation functions, by introducing an appropriate number of arbitrary vectors $\mathbf{a}, \mathbf{b}, \mathbf{c}, \ldots$, etc.

The procedure we will follow here can be used to find the correlation between two turbulent fields; these fields may be two vector fields, one scalar and one vector field or two scalar fields.

\section{Bilinear form}

Returning to the correlation function in Eq. (B1) we notice that it is possible to build two different correlation functions $R_{i j}$; 


$$
R_{i j}^{(1)}\left(\mathbf{x}, t ; \mathbf{x}^{\prime}, t^{\prime}\right)=\left\langle u_{i}(\mathbf{x}, t) v_{j}\left(\mathbf{x}^{\prime}, t^{\prime}\right)\right\rangle
$$

and

$$
R_{i j}^{(2)}\left(\mathbf{x}, t ; \mathbf{x}^{\prime}, t^{\prime}\right)=\left\langle v_{i}(\mathbf{x}, t) u_{j}\left(\mathbf{x}^{\prime}, t^{\prime}\right)\right\rangle .
$$

The superscripts (1) or (2) refers to the ordering of $\mathbf{u}$ and $\nabla$. From the equality

$$
\left\langle\mathbf{a} \cdot \mathbf{u}(\mathbf{x}, t) \mathbf{b} \cdot \mathbf{v}\left(\mathbf{x}^{\prime}, t^{\prime}\right)\right\rangle=\left\langle\mathbf{b} \cdot \mathbf{v}\left(\mathbf{x}^{\prime} t^{\prime}\right) \mathbf{a} \cdot \mathbf{u}(\mathbf{x}, t)\right\rangle
$$

it follows that

$$
R_{i j}^{(1)}\left(\mathbf{x}, t ; \mathbf{x}^{\prime}, t^{\prime}\right)=R_{j i}^{(2)}\left(\mathbf{x}^{\prime}, t^{\prime} ; \mathbf{x}, t\right)
$$

and with the homogeneity and stationarity conditions it gives

$$
R_{i j}^{(1)}(\xi, \tau)=R_{j i}^{(2)}(-\xi,-\tau) .
$$

Let us now build $R_{0}^{(\alpha)}$ as a bilinear form $a_{i} b_{j}$ by using all permissible scalar products, i.e., $\xi \cdot \mathbf{a}, \xi \cdot \mathbf{b}, \xi \cdot \xi, \mathbf{a} \cdot \mathbf{b}, \lambda \cdot \mathbf{a}, \lambda \cdot \mathbf{b}$, and $\lambda \cdot \xi$. It follows that

$$
\begin{aligned}
R_{0}^{(\alpha)}(\xi, \lambda, \mathbf{a}, \mathbf{b})= & A^{(\alpha)}(\xi \cdot \mathbf{a})(\xi \cdot \mathbf{b})+B^{(\alpha)}(\mathbf{a} \cdot \mathbf{b})+C^{(\alpha)} \\
& \times(\lambda \cdot \mathbf{a})(\lambda \cdot \mathbf{b})+D^{(\alpha)}(\xi \cdot \mathbf{a})(\lambda \cdot \mathbf{b}) \\
& +\mathbf{E}^{(\alpha)}(\xi \cdot \mathbf{b})(\lambda \cdot \mathbf{a}),
\end{aligned}
$$

with $\alpha=1$ or 2 . From which

$$
R_{i j}^{(\alpha)}=A^{(\alpha)} \xi_{i} \xi_{j}+B^{(\alpha)} \delta_{i j}+C^{(\alpha)} \lambda_{i} \lambda_{j}+D^{(\alpha)} \xi_{i} \lambda_{j}+E^{(\alpha)} \lambda_{i} \xi_{j},
$$

where $A^{(\alpha)}, B^{(\alpha)}, C^{(\alpha)}, D^{(\alpha)}$, and $E^{(\alpha)}$ are functions of $r^{2}=\xi \cdot \xi, r \mu=\xi \cdot \lambda$, and $\tau$. By imposing reversibility in time, it follows $R_{0}^{(\alpha)}(\xi, \lambda, \mathbf{a}, \mathbf{b},-\tau)=R_{0}^{(\alpha)}(\xi, \lambda, \mathbf{a}, \mathbf{b}, \tau)$ which, in turn, implies that $A^{(\alpha)}, B^{(\alpha)}, \ldots, E^{(\alpha)}$ are even function of $\tau$. Thus, due to homogeneity and cylindrical symmetry, we only have to find five functions to determine completely the nine correlation functions involved in $R_{i j}^{(\alpha)}$. Moreover, as will be shown below, in the cases when $\mathbf{v}=\mathbf{u}$ or the vector fields $u$ and $v$ are divergence free we only have to give four functions instead of five.

From Eqs. (B6) and (B7) it follows

$$
A^{(1)}\left(r^{2}, r \mu\right)=A^{(2)}\left(r^{2},-r \mu\right)
$$

and similarly for $B^{(\alpha)}$ and $C^{(\alpha)}$. The relationships between $D^{(\alpha)}$ and $E^{(\alpha)}$ are given by

$$
D^{(1)}\left(r^{2}, r \mu\right)=-E^{(2)}\left(r^{2},-r \mu\right)
$$

and

$$
E^{(1)}\left(r^{2}, r \mu\right)=-D^{(2)}\left(r^{2},-r \mu\right) .
$$

In the case $\mathbf{v}=\mathbf{u}$ it results $R_{i j}^{(1)}=R_{i j}^{(2)}$ and therefore $A^{(1)}=A^{(2)}, B^{(1)}=B^{(2)}, \ldots, E^{(1)}=E^{(2)}$; this means that $A, B$, and $C$ are even functions in $\mu r$ and $D\left(r^{2}, r \mu\right)$ $=-E\left(r^{2},-r \mu\right)$. Notice from (B6) that $R_{i j}$ is not symmetric in the subscripts.

In what follows, we analyze the case with $\mathbf{u}$ a divergence-free stochastic field with $\partial u_{i} / \partial x_{i}=0$. It follows from Eq. (B3) that

$$
-\frac{\partial R_{i j}^{(1)}}{\partial x_{i}}=\frac{\partial R_{i j}^{(1)}}{\partial \xi_{i}}=\cdots\left\langle\frac{\partial u_{i}(\mathbf{x}, t)}{\partial x_{i}} v_{j}\left(\mathbf{x}^{\prime}, t^{\prime}\right)\right\rangle=0
$$

and

$$
\frac{\partial R_{i j}^{(2)}}{\partial x_{j}^{\prime}}=\frac{\partial R_{i j}^{(2)}}{\partial \xi_{j}}=\left\langle v_{i}(\mathbf{x}, t) \frac{\partial u_{j}\left(x^{\prime}, t^{\prime}\right)}{\partial x_{j}^{\prime}}\right\rangle=0 .
$$

In the case $\mathbf{v}$ is also divergence free it also holds that $\partial R_{i j}^{(1)} / \partial \xi_{j}=\partial R_{i j}^{(2)} / \partial \xi_{i}=0$. Let us now consider Eq. (B11b) and substitute expression (B8) into it to obtain

$$
\begin{gathered}
\xi_{i}\left[4 A^{(2)}+r \frac{\partial A^{(2)}}{\partial r}+\frac{1}{r} \frac{\partial B^{(2)}}{\partial r}-\frac{\mu}{r^{2}} \frac{\partial B^{(2)}}{\partial \mu}+\mu \frac{\partial E^{(2)}}{\partial r}\right. \\
\left.+\frac{\left(1-\mu^{2}\right)}{r} \frac{\partial E^{(2)}}{\partial \mu}\right]+\lambda_{i}\left[\frac{1}{r} \frac{\partial B^{(2)}}{\partial \mu}+\mu \frac{\partial C^{(2)}}{\partial r}+\frac{\left(1-\mu^{2}\right)}{r}\right. \\
\left.\times \frac{\partial C^{(2)}}{\partial \mu}+3 D^{(2)}+r \frac{\partial D^{(2)}}{\partial r}+E^{(2)}\right]=0
\end{gathered}
$$

where we used

$$
\frac{\partial}{\partial \xi_{j}}=\left(\frac{\xi_{j}}{r}\right) \frac{\partial}{\partial r}+\left(\frac{\lambda_{j}}{r}-\frac{\mu \xi_{j}}{r^{2}}\right) \frac{\partial}{\partial \mu} .
$$

Since $\xi$ may be any arbitrary vector, the expressions in square brackets should vanish by themselves. This gives rise to two equations involving the functions $A^{(2)}$, $B^{(2)}, \ldots, E^{(2)}$, from which two functions can be deduced if we specify the other three. If we use Eq. (B11a) we get a similar equation to (B12) with $A^{(1)}, B^{(1)}$, and $C^{(1)}$ instead of those functions with superindex (2), and with $D^{(1)}$ instead $E^{(2)}$ and $E^{(1)}$ instead of $D^{(2)}$.

When, in addition to $\mathbf{u}$ the field $\mathbf{v}$ is also divergence free, we can calculate $\partial R_{i j}^{(2)} / \partial x_{i}=-\partial R_{i j}^{(2)} / \partial \xi_{i}=0$, and obtain an equation similar to (B12); again, since $\xi$ is an arbitrary vector we set each of the two terms in the resulting equation equal to zero and obtain two equations. After subtracting these expressions from the equations obtained from Eq. (B12) we have

$$
\begin{aligned}
& \mu \frac{\partial}{\partial r}\left(E^{(\alpha)}-D^{(\alpha)}\right)+\frac{\left(1-\mu^{2}\right)}{r} \frac{\partial}{\partial \mu}\left(E^{(\alpha)}-D^{(\alpha)}\right)=0, \\
& 2\left(E^{(\alpha)}-D^{(\alpha)}\right)+r \frac{\partial}{\partial r}\left(E^{(\alpha)}-D^{(\alpha)}\right)=0,
\end{aligned}
$$

where $\alpha=1$ or 2 . The solution of the last equation yields $E^{(\alpha)}-D^{(\alpha)}=K(\mu) / r^{2}$, which when substituted in the former one together with the condition that $E^{(\alpha)}$ and $D^{(\alpha)}$ be finite at $r=0$ and at $\mu= \pm 1$, it follows that

$$
E^{(\alpha)}=D^{(\alpha)} .
$$

From Eqs. (B10) and (B14) it turns out that $D$ and $E$ are odd functions in $\mu$ in the case when $\mathbf{u}=\mathbf{v}$. Without loss of generality, we can choose a coordinate system with the $z$ axis in direction of $\lambda$ and write $R_{i j}$ in a symmetric matrix form as 
$R_{i j}=\left(\begin{array}{ccc}A \xi_{x}^{2}+B & A \xi_{x} \xi_{y} & A \xi_{x} \xi_{z}+D \xi_{x} \\ A \xi_{y} \xi_{x} & A \xi_{y}^{2}+B & A \xi_{y} \xi_{z}+D \xi_{y} \\ A \xi_{z} \xi_{x}+D \xi_{x} & A \xi_{z} \xi_{y}+D \xi_{y} & A \xi_{z}^{2}+B+C+2 \xi_{z} D\end{array}\right)$

when the correlation is taken at the same point, i.e., $\xi=0$, $R_{i j}$ becomes a diagonal matrix.

\section{Linear form}

Let us now consider the correlation

$$
L_{0}(\xi, \lambda, \mathbf{a}, \tau)=\left\langle\Phi(\mathbf{x}, t) u_{i}\left(\mathbf{x}^{\prime}, t^{\prime}\right) a_{i}\right\rangle,
$$

with $a_{i}$ the $i$ th component of an arbitrary vector $\mathbf{a}$, and $\Phi$ and $\mathbf{u}$ are turbulent scalar and vector fields, respectively. Thus we have

$$
L_{0}(\xi, \lambda, a, \tau)=L_{i}(\xi, \lambda, \tau) a_{i} .
$$

The scalar products that can be formed are $(\xi \cdot a),(\xi \cdot \lambda)$, $(\xi \cdot \xi),(\lambda \cdot a),(a \cdot a)$, and $(\lambda \cdot \lambda)=1$. In order to build a linear function of a we should take

$$
L_{0}(\xi, \lambda, \mathbf{a}, \tau)=M(\xi \cdot \mathbf{a})+N(\lambda \cdot \mathbf{a}),
$$

where $M$ and $N$ are functions of $r^{2}=\xi \cdot \xi, r \mu=\xi \cdot \lambda$, and $\tau$. It yields

$$
L_{i}=M \xi_{i}+N \lambda_{i} .
$$

Again we can form two different functions $L_{0}$, i.e.,

$$
L_{0}^{(1)}\left(\mathbf{x}, t ; \mathbf{x}^{\prime}, t^{\prime}\right)=\left\langle u_{i}(\mathbf{x}, t) \Phi\left(\mathbf{x}^{\prime}, t^{\prime}\right)\right\rangle a_{i}
$$

and

$$
L_{0}^{(2)}\left[\mathbf{x}, t ; \mathbf{x}^{\prime}, t^{\prime}\right]=\left\langle\Phi(\mathbf{x}, t) u_{i}\left(\mathbf{x}^{\prime}, t^{\prime}\right)\right\rangle a_{i} ;
$$

this means

$$
L_{i}^{(\alpha)}=M^{(\alpha)} \xi_{i}+N^{(\alpha)} \lambda_{i},
$$

with $\alpha=1$ or 2 . As by Eq. (B6), when homogeneity and stationarity conditions are imposed, it holds that

$$
L_{0}^{(1)}(\xi, \tau)=L_{0}^{(2)}(-\xi,-\tau)
$$

and, assuming time reversibility, we obtain

$$
L_{0}^{(\alpha)}(\xi,-\tau)=L_{0}^{(\alpha)}(\xi, \tau) .
$$

This second condition means that $M^{(\alpha)}$ and $N^{(\alpha)}$ are even functions of $\tau$ and $\mathrm{Eq}$. (B22) yields

$$
M^{(1)}\left(r^{2}, r \mu, \tau\right)=-M^{(2)}\left(r^{2},-r \mu, \tau\right)
$$

and

$$
N^{(1)}\left(r^{2}, r \mu, \tau\right)=N^{(2)}\left(r^{2},-r \mu, \tau\right) .
$$

When $\nabla \cdot \mathbf{u}=0$ it follows that

$$
\frac{\partial L_{i}^{(\alpha)}}{\partial \xi_{i}}=0,
$$

which gives a relationship between $M^{(\alpha)}$ and $N^{(\alpha)}$.

\section{Scalar form}

Finally, let us consider the correlation between two stochastic scalar functions, say $\Phi(\mathbf{x}, t ; \widetilde{\omega})$ and $\Psi\left(\mathbf{x}^{\prime}, t^{\prime} ; \widetilde{\omega}\right)$ to build the following:

$$
\begin{aligned}
& F^{(1)}\left(\mathbf{x}, t ; \mathbf{x}^{\prime} t^{\prime}\right)=\left\langle\Phi(\mathbf{x}, t) \Psi\left(\mathbf{x}^{\prime}, t^{\prime}\right)\right\rangle, \\
& F^{(2)}\left(\mathbf{x}, t ; \mathbf{x}^{\prime} t^{\prime}\right)=\left\langle\Psi(\mathbf{x}, t) \Phi\left(\mathbf{x}^{\prime}, t^{\prime}\right)\right\rangle,
\end{aligned}
$$

again, with homogeneity and stationarity conditions, we obtain

$$
F^{(1)}(\xi, \tau)=F^{(2)}(-\xi,-\tau)
$$

Due to the cylindrical symmetry it follows that $F^{(r)}(\xi, \tau)$ must depend only on $\xi \cdot \xi$ and $\xi \cdot \lambda$ and, assuming time reversibility, $F^{(\alpha)}$ must be an even function of $\tau$.

In the case $\Psi=\Phi$ it results that $F^{(1)}=F^{(2)}$ and it therefore is an even function of $\xi \cdot \lambda$.

${ }^{1}$ P. Liewer, Nucl. Fusion 25, 543 (1985) and references therein.

${ }^{2}$ A. J. Wootton, B. A. Carreras, H. Matsumoto, K. MeGuire, W. A. Peebles, Ch. P. Ritz, P. W. Terry, and S. J. Zweber, Phys. Fluids B 2, 2879 (1990).

${ }^{3}$ Y. J, Kim, K. W. Gentle, Ch. P. Ritz, T. L. Rhodes, and R. D. Bengtson, Phys. Fluids B 3, 674 (1991).

${ }^{4}$ F. A. Haas and A. Thyagaraja, Phys. Rep, 143, 242 (1986).

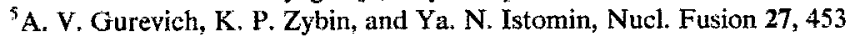
(1987).

${ }^{6}$ A. B. Rechester and M. N. Rosenbluth, Phys. Rev. Lett. 40, 38 (1978).

${ }^{7}$ B. B. Kadomtsev and O. P. Pogutse, in Plasma Physics and Controlled Nuclear Fusion (International Atomic Energy Agency, Vienna, 1979), Vol. 1, p. 649.

${ }^{8}$ J. A. Krommes, C. Oberman, and R. G. Kleva, J. Plasma Phys, 30, 11 (1983).

${ }^{9}$ M. B. Isichenko, Plasma Phys. Controlled Fusion 33, 795 (1991).

${ }^{10}$ R. G. Kleva and J. T. Drake, Phys. Fluids 27, 1686 (1984).

"J. H. Misgui'ch, R. Balescu, H. L. Pe'cseli, T. Mikkelsen, S. E. Larsen, and $Q$. Xiaoming, Plasma Phys. Controlled Fusion 29, 825 (1987).

${ }^{12}$ V. V. Parail and P. N. Yushmanov, JETP Lett. 42, 343 (1985).

${ }^{13}$ W. Horton, D. I. Choi, P. N. Yushmanov, and V. V. Parail, Plasma Phys. Controlled Fusion 29, 901 (1987).

${ }^{14}$ A. Thyagaraja and F. A. Haas, Plasma Phys. Controlled Fusion 31, 965 (1989) and Plasma Phys. Controlled Fusion 31, 1797 (1989).

${ }^{15}$ A. I. Morozov and L. S. Solov'ev, Review of Plasma Physics, edited by M. A. Leontovich (Consultants Bureati, New York, 1966), Vol. 2

${ }^{16}$ N. N. Bogoliubov and Y. A. Mitropolski, Asymptotic Methods in the Theory of Non-Linear Oscillations (Hindustan, Delhi, 1961).

${ }^{17}$ P. A. Duperrex, Ch. Hollenstein, B. Joye, R. Keller, J. B. Lister, F. B. Marcus, J. M. Moret, A. Pochelon, and W. Simm, Phys. Lett. A 106, $133(1984)$

${ }^{18}$ S. J. Zweben and R. J. Taylor, Nucl. Fusion 23, 513 (1983).

${ }^{19}$ G. A. Hallock, J. Mathew, W. C. Jennings, R, L. Hickok, A. J. Wootton, and R. C. Isler, Phys. Rev. Lett. 56, 1248 (1986).

${ }^{20}$ T. I. Bugaraya, A. V. Gorshkov, S. A. Grashin, I. V. Ivanov, V. A. Krupin, A. V. Mel'nikov, K. A. Razumova, Yu. A. Sokolov, V. M. Trukhin, A. V. Chankin, Y. N. Yushmanov, L. I. Krupnik, and I. S. Nedzel'skij, Nucl Fusion 25, 1707 (1985)

${ }^{21}$ P. M. Schoch, R. L. Hickok, and W. C. Jennings, Phys. Rev. Lett. 55, 2417 (1985).

${ }^{22}$ G. A. Hallock, A. J. Wootton, and R. L. Hickok, Phys. Rev. Lett. 59, 1301 (1987).

${ }^{23}$ S. J. Zweben and R. W. Gould, Nucl. Fusion 25, 171 (1985).

${ }^{24}$ S. J. Levinson, J. M. Beall, E. J. Powers, and R. D. Bengtson, Nucl. Fusion 24, 527 (1984).

${ }^{25}$ A. Thyagaraja, J. L. Robertson, and F. A. Haas, Plasma Phys. Controlled Fusion 27, 1217 (1985). 
${ }^{26}$ N. G. Van Kampen, Stochastic Processes in Physics and Chemistry (North-Holland, Amsterdam, 1981).

${ }^{27}$ R. Kubo, J. Math. Phys. 4, 174 (1963).

${ }^{28}$ R. Kubo, J. Phys. Soc. Jpn. 17, 1100 (1962).

${ }^{29}$ E. A. Novikov, Sov. Phys. JETP 20, 1290 (1965).

${ }^{30}$ A. Papoulis, Probability, Random Variables, and Stochastic Processes
(McGraw-Hill, New York, 1984), p. 215.

${ }^{31}$ P. Hanggi, in Stochastic Processes Applied to Physics, edited by $\mathbf{L}$. Pesquera and M. A. Rodriguez (World Scientific, Singapore, 1985).

${ }^{32}$ G. K. Bachelor, Proc. R. Soc. London Ser. A 186, 480 (1946).

${ }^{33} \mathrm{G}$. K. Bachelor, The Theory of Homogeneous Turbulence (Cambridge U.P., Cambridge, 1960). 